\title{
Synthesis of novel 1,2-bis-quinolinyl-1,4-naphthoquinones : ERK2 inhibition, cytotoxicity and molecular docking studies
}

\section{Aly, Ashraf A.}

2018-12

Aly , A A , El-Sheref , E M , Bakheet, M E M , Mourad, M A E , Brown , A B , Bräse , S , Nieger , M \& Ibrahim , M A A 2018 , ' Synthesis of novel

1,2-bis-quinolinyl-1,4-naphthoquinones : ERK2 inhibition, cytotoxicity and molecular docking studies ' , Bioorganic Chemistry , vol. 81 , pp. 700-712 . https://doi.org/10.1016/j.bioorg.2018.09.017

http://hdl.handle.net/10138/322150

https://doi.org/10.1016/j.bioorg.2018.09.017

cc_by_nc_nd

acceptedVersion

Downloaded from Helda, University of Helsinki institutional repository.

This is an electronic reprint of the original article.

This reprint may differ from the original in pagination and typographic detail.

Please cite the original version. 


\section{Graphical Abstract}

\section{Synthesis of novel 1,2-bis-quinolinyl-1,4-naphthoquinones: Targeting by ERK2, cytotoxicity and molecular docking}

\section{Ashraf A. Aly, Essmat M. El-Sheref, Momtaz E. M. Bakheet,}

Mai A. E. Mourad, Alan B. Brown, Stefan Bräse, Tamer S. Kaoud,

Martin Nieger and Mahmoud A. A. Ibrahim<smiles>[R]c1ccc2c(c1)c(O)cc(=O)n2[R1]</smiles><smiles></smiles>

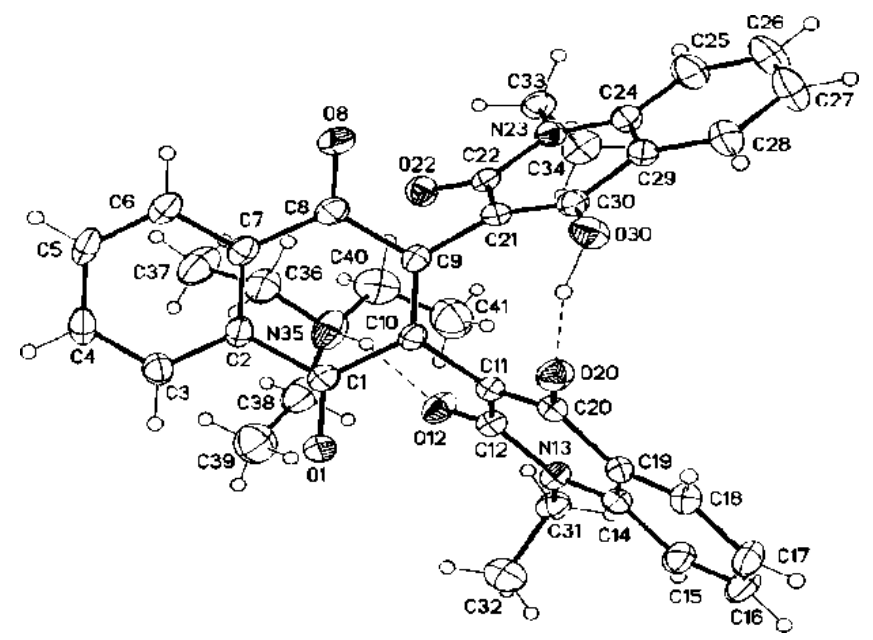

X-ray structural analysis of $\mathbf{4 c}$ (displacement parameters are drawn at 50\% probability level 


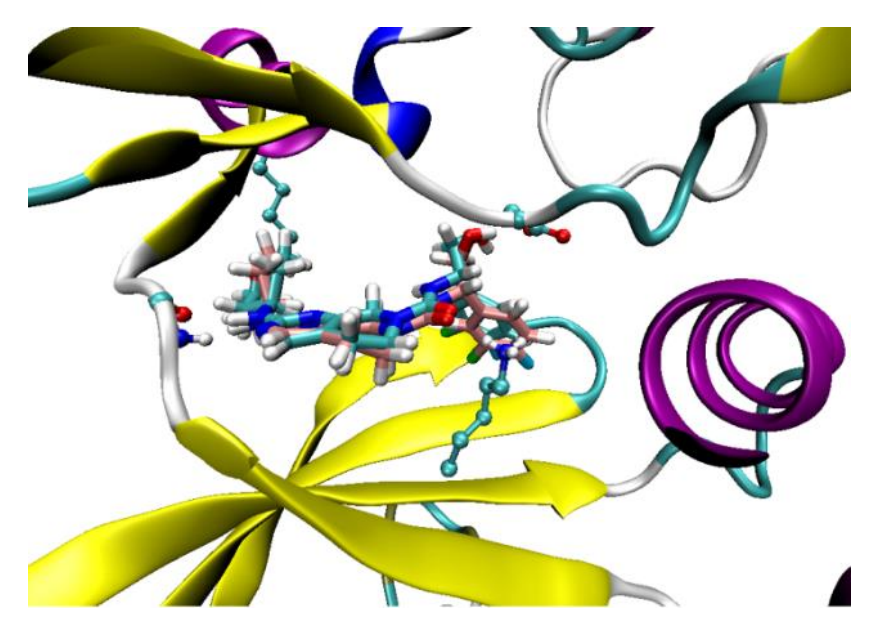

Cartoon backbone representation of crystal structure binding mode (in cyan) and predicted docking pose (in pink) of $2 \mathrm{SH}$ inhibitor with the ERK2 receptor. 


\title{
Synthesis of novel 1,2-bis-quinolinyl-1,4-naphthoquinones: Targeting by ERK2, cytotoxicity and molecular docking
}

\author{
Ashraf A. Aly, ${ }^{1 *}$ Essmat M. El-Sheref, ${ }^{1}$ Momtaz E. M. Bakheet, ${ }^{1}$ Mai A. E. Mourad, ${ }^{2}$ \\ Alan B. Brown, ${ }^{3}$ Stefan Bräse, ${ }^{4}$ Tamer S. Kaoud, ${ }^{5}$ \\ Martin Nieger, ${ }^{6}$ and Mahmoud A. A. Ibrahim ${ }^{1}$
}

${ }^{1}$ Chemistry Department, Faculty of Science, Minia University, 61519-El-Minia, Egypt.

${ }^{2}$ Medicinal Chemistry Department, Faculty of Pharmacy, Port-Said University, Port-Said 42526, Egypt.

${ }^{3}$ Chemistry Department, Florida Institute of Technology, Melbourne, FL 32901, USA

${ }^{4}$ Institute of Organic Chemistry, Karlsruhe Institute of Technology, 76131 Karlsruhe, Germany.

${ }^{5}$ Division of Medicinal Chemistry, The University of Texas at Austin, TX, USA

${ }^{6}$ Department of Chemistry, University of Helsinki, P.O. Box55 (A. I. Virtasen aukio 1), 00014, Helsinki, Finland

Corresponding Author: ashrafaly63@yahoo.com and ashraf.shehata@mu.edu.eg Postal address: Chemistry Department, Faculty of Science, Minia University, Minia 61519, Egypt.

\section{Highlights}

- Novel series of bis-quinolinylquinones 3a-d and 4a-c were obtained and identified by different spectroscopic techniques.

- Targeting ERK2, compounds 3a, 3b and 4c inhibited ETS-1 phosphorylation by ERK2 in a dose dependent manner.

- Molecular docking studies were carried out to explore the binding pattern of some of the newly synthesized compounds towards ATP-binding site of ERK2 receptor.

- Compound 4c showed selectivity for ERK2 inhibition with ATP-competitive inhibition mechanism and further structure improvement for enhancing its pharmacokinetic profile is recommended. 
- In vitro anticancer activity against the NCI-60 panel of tumor cell lines revealed that the tested compounds demonstrated from mild to strong cytotoxic activity.

- The cytotoxic effect of compound 3d was apparent across all tumor cell lines tested, except leukemia, with 47 out of the 52 non-leukemic cell lines showing negative growth percentages.

- NCI in vitro five dose testing revealed that compounds $\mathbf{3 b}$ and $\mathbf{3 d}$ represent promising lead anticancer compounds with $\mathrm{GI}_{50}$ values ranging between $(0.31-3.04 \mu \mathrm{M})$, and $(0.26$ $-0.93 \mu \mathrm{M})$, respectively.

\begin{abstract}
In an effort to establish new candidates targeting ERK2 with improved antineoplastic activity, two novel series of $N$-2,3-bis(6-substituted-4-hydroxy-2-oxo-1,2dihydroquinolin-3-yl)naphthalene-1,4-diones 3a-d and $\quad$ substituted $N$ (methyl/ethyl)bisquinolinone triethylammonium salts $\mathbf{4 b , c}$ were successfully synthesized. The synthesis involved the reactions of 2 equivalents of 4-hydroxy-2(1H)-quinolinones 1a-f and one equivalent of 1,4-naphthoquinone (2) in a mixture of ethanol/dimethylformamide $(1: 1)$ as a solvent and $0.5 \mathrm{ml} \mathrm{Et}_{3} \mathrm{~N}$. In case of the reaction of 6-methyl-4-hydroxyquinolone $\mathbf{1 b}$ with $\mathbf{2}$, a side product $\mathbf{4 a}$ of the second series was obtained. In general, the presence of free $\mathrm{NH}$-quinolone gave a single compound of the first series, whereas reaction of $N$-methyl/ethyl-quinolones $\mathbf{1 e}, \mathbf{f}$ with $\mathbf{2}$ enhanced the formation of compounds of the second series. The structures of the new compounds were proved by different spectroscopic techniques such as IR, NMR (2D-NMR) and mass spectra, elemental analysis, and X-ray crystallography. In order to further elucidate the mechanism of action of these newly synthesized compounds, compounds $\mathbf{3 a}, \mathbf{3 b}, \mathbf{4 b}$ and
\end{abstract}


$4 \mathbf{c}$ were investigated for their MAP Kinase pathway inhibition together with molecular docking using ATP-binding site of ERK2 receptor. The results revealed that compounds 3a, 3b and 4c inhibited ETS-1 phosphorylation by ERK2 in a dose dependent manner. Also, compound $\mathbf{4 c}$ showed highest potency and selectivity for ERK2 inhibition with ATP-competitive inhibition mechanism which was confirmed by the formation of three hydrogen bond in the molecular docking studies. Interestingly, the synthesized compounds were then tested for their in vitro anticancer activity against the NCI-60 panel of tumor cell lines. The results revealed that the selected compounds displayed from modest to strong cytotoxic activities. Compound $\mathbf{3 b}$ demonstrated broad spectrum antitumor activity against the nine tumor sub-panels tested, while compound $\mathbf{3 d}$ proved to be lethal to the majority of the cancer lines which was approved by their promising $\mathrm{GI}_{50}$ and TGI values in NCI in vitro five dose testing. These results revealed that the synthesized compounds can potentially serve as leads for the development of novel chemotherapeutic agents and structure improvement will be necessary for some derivatives for enhancing its cellular activities and pharmacokinetic profile.

Keywords: 1,2-Bis-quinolinyl-1,4-naphthoquinones; 1,4-Naphthoquinone; Cytotoxicity; Targeting by ERK2; Molecular docking.

\section{Introduction}

Mitogen activated protein kinase (MAPK) is probably considered the major signaling transduction pathway in cell biology which plays a pivotal role in the regulation of multiple cell

processes [1]. MAPK pathway is implicated in the regulation of gene expression, apoptosis, metabolism, cell proliferation and differentiation [2]. Indeed, MAPK appears to induce tumor growth, angiogenesis and metastasis by dysregulation of three major pathways which are ERK, JNK and p38 [3]. ERK2 inhibition attracted a great interest within the ERK kinase subgroup since 
this protein can have cell pro-proliferative effects. In this light, a number of studies have been performed for the development of ERK inhibitors for human cancer treatment [4]. The quinone scaffold is present in many clinically used cancer chemotherapeutic drugs $[1,2]$. Furthermore, 1,4naphthoquinones are used widely in the pharmaceutical and agrochemical industries. They possess a variety of pharmacological properties such as anticancer [3-6], antimicrobial [7,8], antiviral [9-11], antifungal [12-15], and antiplatelet activities [16-18]. Folkers et al. [19] reported that 1,4-benzoquinone derivatives - the analogs of coenzyme Q10 - are potent compounds in an antitumor test system in vivo, in addition to their effective antitumor activity in other tumor systems. 2-Phenyl-3-hydroxyquinolin-4(1H)-ones exhibit pronounced biological activities and have been identified as strong anticancer agents with significant in vitro cytotoxicity against various cancer cell lines [20-23]. Bis-quinolones of type I (Figure 1) occur naturally, and have fluorescent properties and biological activity [24,25]. In a recent communication [26], the synthesis of the zwitter-ionic 4-hydroxycoumarin derivatives II (Figure 1) was reported, through a unique reaction of 4-hydroxycoumarins with $p$-benzoquinone and pyridine in aqueous acetone. A recent report shows the utility of 4-hydroxy-1-phenylquinolin-2(1H)-one in a one-pot synthesis of pyrimidoquinolines [27]. We reacted 4-hydroxy-1-phenylquinolin-2(1H)-one with diethyl acetylenedicarboxylate, to give ethyl 5,6-dihydro-2,5-dioxo-6,9-disubstituted-2H-pyrano[3,2c]quinoline-4-carboxylates in good yields [28]. We also synthesized 2'-amino-2,5'-dioxo-5',6'dihydro-spiro(indoline-3,4'-pyrano[3,2-c]-quinoline)-3'-carbonitriles in good to excellent yields from the reactions of 4-hydroxy-1-phenylquinolin-2(1H)-one with 2-(2-oxo-1,2-dihydroindol-3ylidene)-malononitrile [29]. In view of the aforesaid and the expected anticancer activity of the above pharmacophores, we now report the design of the novel title compounds as a combination of the most active quinolinone and naphthoquinone entities. ERK2 inhibition mechanism towards some of the synthesized compounds was studied. Meanwhile, molecular docking study for the synthesized compounds with ATP-binding site of ERK2 receptor was carried out to reveal the inhibitor-receptor binding mode. Also, the cytotoxicity of the synthesized compounds was 
evaluated against NCI 60 cell lines of different nine tumor subpanels. Moreover, compounds $\mathbf{3 b}$ and 3d were further selected towards NCI in vitro five dose testing.

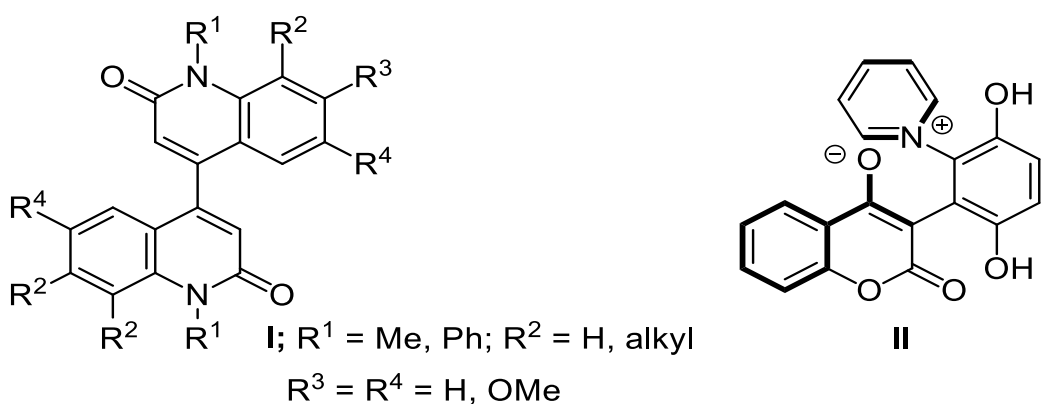

Figure 1. Structures of bis-quinolones I and Zwitterionic salt of quinolone II

\section{Experimental Section}

\subsection{Biological investigation}

\section{Targeting ERK2 inhibition:}

In order to study the mechanism of action of the newly synthesized compounds, compounds $\mathbf{3 a}, \mathbf{3 b}, \mathbf{4 b}$ and $\mathbf{4 c}$ were investigated for MAP Kinase pathway inhibitory activity. The results revealed that treatment of $B R A F$ - mutant A375 melanoma cell lines with the tested compounds 3a, 3b and 4c inhibited Ets-1 phosphorylation by ERK2 in a dose dependent manner (Table 1 and Fig. 4). In addition, the results recorded in Table 1 indicated that compound 4c potently inhibited ERK2 activity with $\mathrm{IC}_{50}$ value of $0.71 \mu \mathrm{M}$. While, compounds $\mathbf{3 a}$ and $\mathbf{3 b}$ inhibited ERK2 activity with $\mathrm{IC}_{50}$ values of $10.68 \mu \mathrm{M}$ and $8.65 \mu \mathrm{M}$, respectively. Moreover, compound $4 \mathbf{c}$ inhibited phosphorylation by JNK and $\mathrm{P} 38 \alpha$ at $\mathrm{IC}_{50}$ values of $10.5 \mu \mathrm{M}$ and 10.2 $\mu \mathrm{M}$, respectively. This in turn, indicated the selectivity of compound $\mathbf{4 c}$ towards ERK2 inhibitory activity which may be attributed to the formation of three hydrogen bonds with Asp111, Ser153 and Cys166 in the ATP-binding site of ERK2 receptor (as illustrated in molecular docking calculations). Also, Fig. 4 (E) demonstrated that the ability of compound $4 \mathbf{c}$ to inhibit Ets-1 phosphorylation by ERK2 is decreased by increasing concentrations of ATP which suggesting ATP-competitive inhibition mechanism. 
Table 1. The ability of the tested compounds to inhibit MAP kinases activity

\begin{tabular}{ccccc}
\hline & $\begin{array}{c}\text { 3a } \\
\mathrm{IC}_{50}{ }^{a}(\mu \mathrm{M})\end{array}$ & $\begin{array}{c}\text { 3b } \\
\mathrm{IC}_{50}{ }^{a}(\mu \mathrm{M})\end{array}$ & $\begin{array}{c}\mathbf{4 b} \\
\mathrm{IC}_{50}{ }^{a}(\mu \mathrm{M})\end{array}$ & $\begin{array}{c}\mathbf{4 c} \\
\mathrm{IC}_{50}{ }^{a}(\mu \mathrm{M})\end{array}$ \\
\hline ERK2 & $10.68 \pm 1.2$ & $8.65 \pm 1.4$ & $>100$ & $0.71 \pm 0.1$ \\
JNK2 & $>100$ & $94 \pm 10.2$ & $>100$ & $10.5 \pm 1.4$ \\
p38 $\alpha$ & $>100$ & $89 \pm 9.1$ & $>100$ & $10.2 \pm 1.3$ \\
\hline
\end{tabular}

${ }^{\mathrm{a}} \mathrm{IC}_{50}$ Determined as described in Methods section, Data were fitted to equation $\mathbf{1}$.

A.

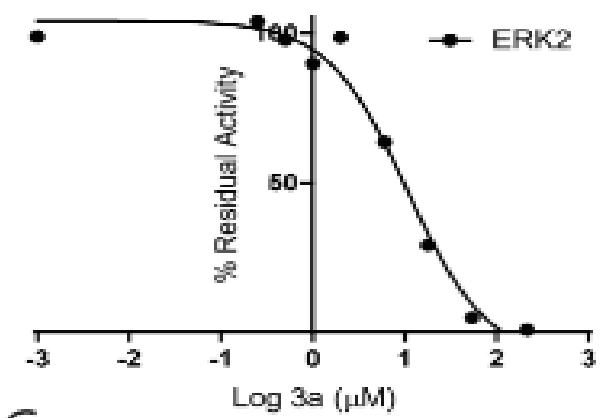

C.

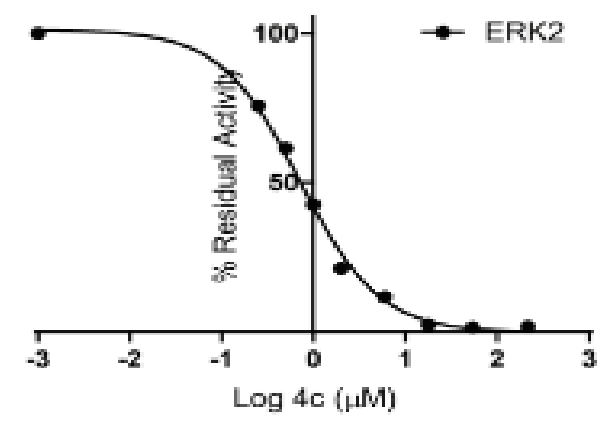

E.

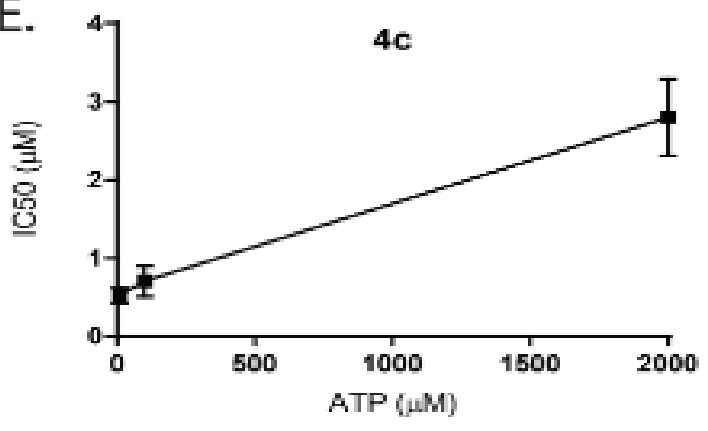

B.

D.
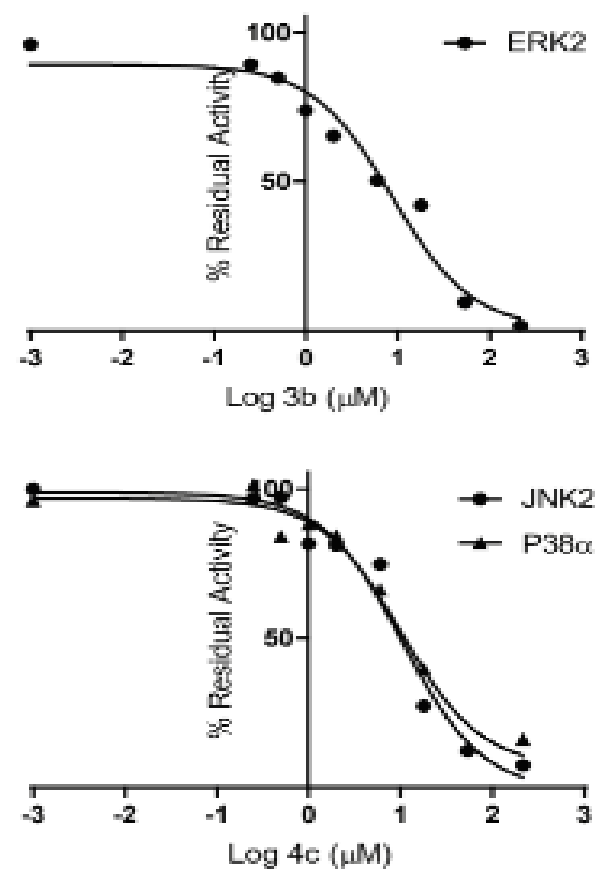
Figure 1. The effect of compounds 3a, $\mathbf{3 b}$ and $\mathbf{4 c}$ on the MAP kinases activity in vitro. In an in -vitro kinase assays, 3a (A) $3 \mathbf{b}$ (B) and $4 \mathbf{c}$ (C) inhibited Ets-1 phosphorylation by ERK2 in a dose dependent manner. (D) Compound 4c showed less potency in inhibiting GST-c-Jun (1-221) phosphorylation by JNK2 and GSTATF2 (1 -115) phosphorylation by P38 $\alpha$ if compared to its ability to inhibit ERK2 kinase activity. Data were fitted to Eq. 1 (E) Higher concentrations of ATP decreased the ability of compound $\mathbf{4 c}$ to inhibit Ets-1 phosphorylation by ERK2 suggesting ATP-competitive inhibition mechanism.

\subsection{Molecular docking calculations}

To reveal the binding features of the synthesized compounds with ERK2 receptor, molecular docking calculations were performed. Docking calculations were carried out on the top potent compounds towards the ATP-active site of ERK2 receptor (PDB code: 4O6E) using Autodock4.2 software. Self-docking of the original ligand (i.e. $2 \mathrm{SH}$ ) with ERK2 receptor was first carried out to assess the performance of Autodock in predicting the ligand-receptor binding mode. Self-docking calculation indicated excellent performance of Autodock software with RMSD value of $0.25 \AA$ (Figures 5 and $6)$. 


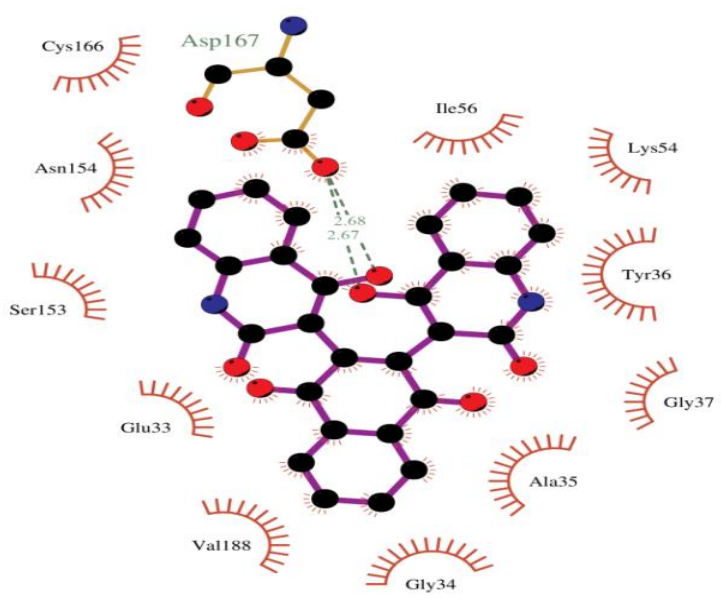

(i)

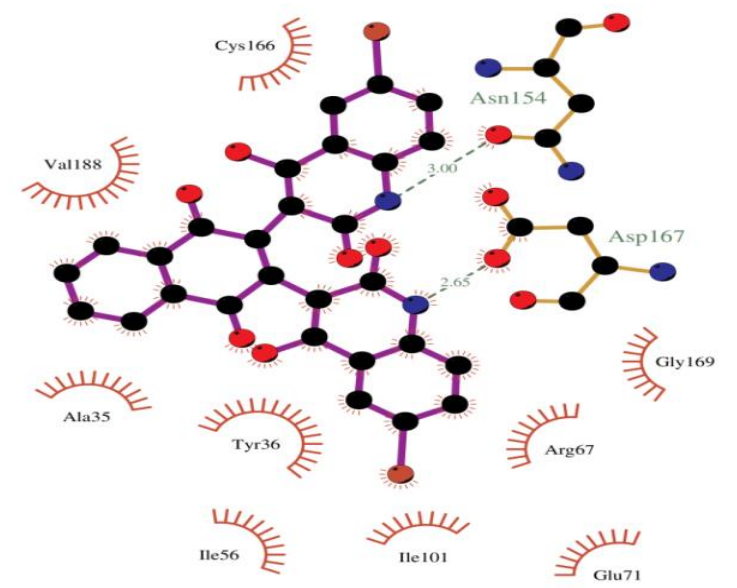

(iii)

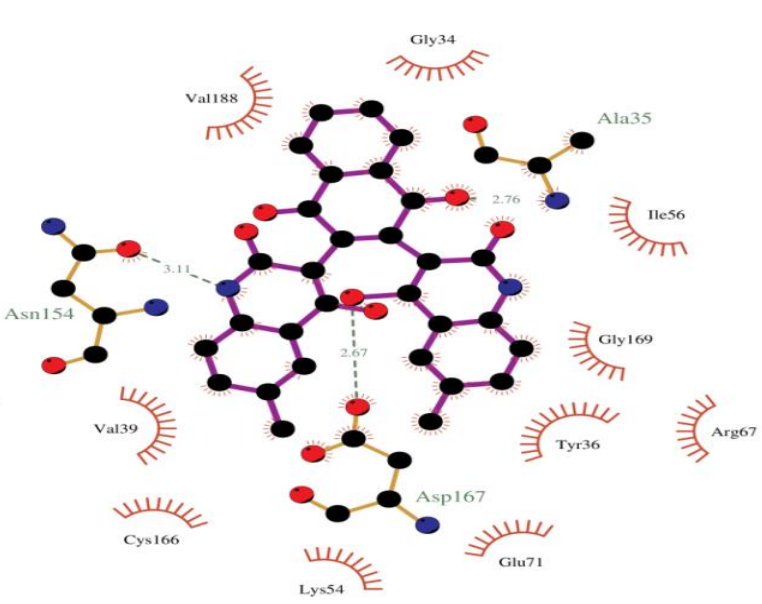

(ii)

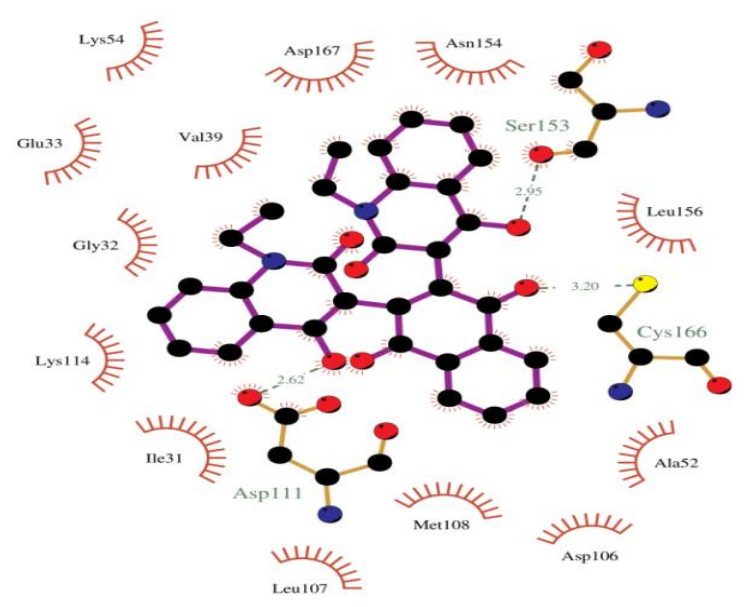

(iv)

Figure 5. 2D Ligplus representation of (i) 3a, (ii) $\mathbf{3 b}$, (iii) $\mathbf{3 d}$, and (iv) $\mathbf{4 c}$ with important amino acid residues of ATP-binding site of ERK2 receptor.

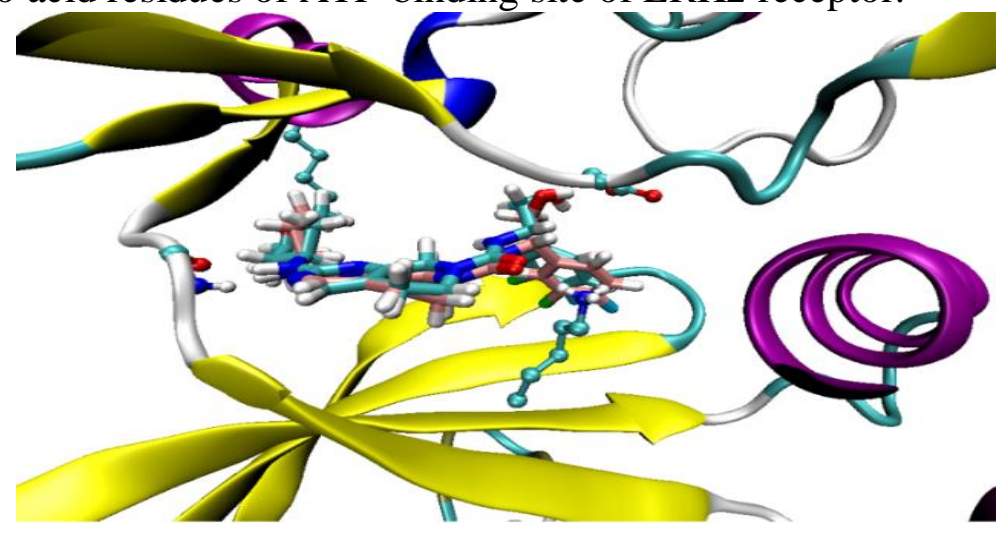

Figure 6. Cartoon backbone representation of crystal structure binding mode (in cyan) and predicted docking pose (in pink) of 2SH inhibitor with the ERK2 receptor. 
Molecular docking calculations were then performed for the synthesized compounds 3a, $\mathbf{3 b}, \mathbf{3 d}$ and $\mathbf{4 c}$ towards ATP-active site of ERK2 receptor. The binding features of these compounds were then investigated. 2D representations of the binding modes of the examined compounds inside the ATP-binding site of ERK2 receptor are depicted in Figure 5. According to the docking results, compounds $\mathbf{3 a}, \mathbf{3 b}$ and $\mathbf{3 d}$ showed similar binding modes and affinities with ERK2 receptor. The calculated binding energies were 8.65, -8.62 and $-8.84 \mathrm{kcal} / \mathrm{mol}$ for compounds $\mathbf{3 a}, \mathbf{3 b}$ and $\mathbf{3 d}$, respectively, with ERK2 receptor. The analysis of binding mode showed that compound 3a forms two essential hydrogen bonds with lengths of 2.68 and $2.67 \AA$ with carboxylate group of Asp167 amino acid. Similarly, compounds $\mathbf{3 b}$ and $\mathbf{3 d}$ form an essential hydrogen bond with Asp167 with bond length of 2.67 and $2.65 \AA$, respectively. While, substitution of $\mathrm{R}^{2}$ with a methyl group and bromine atom in compounds $\mathbf{3 b}$ and $\mathbf{3 d}$, respectively, resulted in formation of a hydrogen bond between the $\mathrm{N}$-quinolin atom and carboxylate group of Asn154 with bond length of 3.11 and $3.00 \AA$, respectively. Compared to compound 3 derivatives, compound $\mathbf{4 c}$ showed better affinity towards ERK2 with binding energy of $9.63 \mathrm{kcal} / \mathrm{mol}$. The better affinity of $\mathbf{4 c}$ may be attributed to the formation of three hydrogen bonds with Asp111, Ser153 and Cys166 with bond lengths of 2.62, 2.95 and $3.20 \AA$ A compared to two hydrogen bonds for compound 3 derivatives. The molecular docking results could support the postulation that our synthesized compounds may act as potent ERK2 inhibitors.

\subsubsection{In vitro anticancer screening at $N C I$}

A series of new fused dihydroxyisoquinolin-naphthalene-1,4-diones were synthesized and submitted to Development Therapeutic Program, National Cancer Institute (NCI), Chemotherapeutic Research division, USA, for evaluation of their in vitro antitumor activities. 
Compounds 3a-d and 4b,c were selected and evaluated against 60 cell lines which included nine tumor subpanels (leukemia, melanoma, lung, colon, CNS, ovarian, renal, prostate and breast cancers). Potential anticancer effect of the synthesized compounds at single concentration of $10^{-5}$ $M$ has been reported as the graph of mean growth percentage $(\mathrm{G} \%)$ of all the treated sixty cell lines compared to untreated control cells which give both inhibition values (between 0 and 100) and cytotoxicity values (less than 0 ).

Table 1. Growth percent (G \%) of the NCI-60 panel tumor cell lines at $10^{-5} \mathrm{M}$ concentration of compounds 3a-d, and $\mathbf{4 b , c}$

\begin{tabular}{|c|c|c|c|c|c|c|}
\hline \multicolumn{2}{|c|}{ Subpanel tumor cell lines } & \multicolumn{5}{|c|}{$\%$ Growth Percent (G \%) } \\
\hline & $\mathbf{3 a}$ & $3 \mathbf{b}$ & $3 \mathbf{c}$ & 3d & $4 \mathbf{b}$ & $4 c$ \\
\hline \multicolumn{7}{|l|}{ Leukemia } \\
\hline CCRF-CEM & 157.34 & 16.64 & 123.82 & 25.33 & 154.72 & 115.36 \\
\hline HL-60(TB) & 126.94 & 8.93 & 115.05 & 45.57 & 173.59 & 139.37 \\
\hline $\mathrm{K}-562$ & 107.83 & 10.70 & 101.76 & 14.32 & 127.16 & 112.87 \\
\hline MOLT-4 & 100.16 & -13.27 & 119.44 & 30.95 & 123.74 & 109.37 \\
\hline RPMI-8226 & 111.83 & 32.81 & 107.40 & 6.40 & 112.10 & 106.00 \\
\hline SR & 102.03 & 23.12 & 125.95 & 20.78 & 123.68 & 99.16 \\
\hline \multicolumn{7}{|c|}{ Non-Small Cell Lung Cancer } \\
\hline A549/ATCC & 106.51 & 21.84 & 106.57 & -32.20 & 102.98 & 102.69 \\
\hline EKVX & ND & ND & ND & ND & ND & ND \\
\hline HOP-62 & 90.58 & 8.69 & 97.16 & -52.85 & 92.57 & 98.12 \\
\hline HOP-92 & 104.40 & 38.67 & 101.81 & -13.17 & 115.72 & 107.30 \\
\hline NCI-H226 & 94.53 & -1.84 & 111.49 & -30.41 & 96.43 & 103.94 \\
\hline NCI-H23 & 80.95 & 15.52 & 92.32 & -43.00 & 90.46 & 97.55 \\
\hline NCI-H322M & 86.05 & 38.80 & 95.01 & -3.29 & 89.13 & 86.60 \\
\hline NCI-H460 & 93.39 & -39.82 & 110.78 & -80.88 & 109.24 & 109.68] \\
\hline NCI-H522 & 104.91 & 7.58 & 88.89 & -31.36 & 96.60 & 101.92 \\
\hline \multicolumn{7}{|l|}{ Colon Cancer } \\
\hline COLO 205 & 98.03 & 69.95 & 111.13 & 73.06 & 104.66 & 105.28 \\
\hline HCC-2998 & 95.32 & 62.03 & 92.50 & -44.34 & 95.91 & 103.12 \\
\hline HCT-116 & 94.46 & 12.93 & 104.95 & -21.67 & 112.33 & 102.96 \\
\hline HCT-15 & 94.52 & 13.71 & 104.45 & -36.33 & 94.96 & 92.28 \\
\hline HT29 & 110.94 & 21.67 & 108.77 & 25.75 & 98.61 & 107.52 \\
\hline KM 12 & 93.44 & 18.46 & 100.50 & -53.20 & 96.45 & 96.96 \\
\hline SW-620 & 93.64 & 7.11 & 105.55 & -70.66 & 107.45 & 99.87 \\
\hline \multicolumn{7}{|l|}{ CNS Cancer } \\
\hline SF-268 & 100.04 & 9.40 & 102.51 & -41.39 & 102.31 & 92.12 \\
\hline SF-295 & 102.40 & 49.86 & 96.29 & 17.19 & 102.81 & 100.28 \\
\hline SF-539 & 100.24 & 61.11 & 104.25 & -57.73 & 102.09 & 99.73 \\
\hline SNB-19 & 96.45 & 26.35 & 104.11 & 4.13 & 98.32 & 103.48 \\
\hline SNB-75 & 94.29 & 14.38 & 91.68 & -35.78 & 111.60 & 100.25 \\
\hline U251 & 101.93 & -16.17 & 109.35 & -57.86 & 106.73 & 98.32 \\
\hline \multicolumn{7}{|l|}{ Melanoma } \\
\hline LOX IMVI & 94.03 & 12.07 & 95.52 & -67.49 & 94.81 & 93.52 \\
\hline MALME-3M & 85.19 & -39.07 & 106.59 & -74.34 & 100.38 & 87.85 \\
\hline M14 & 98.32 & 35.68 & 108.82 & -31.93 & 102.68 & 96.61 \\
\hline MDA-MB-435 & 99.81 & 30.71 & 106.13 & -63.36 & 108.62 & 102.39 \\
\hline SK-MEL-2 & 107.78 & 11.80 & 101.33 & -14.67 & 98.85 & 113.69 \\
\hline SK-MEL-28 & 108.14 & 34.91 & 108.43 & -47.84 & 103.70 & 104.43 \\
\hline SK-MEL-5 & 96.30 & 25.84 & 108.92 & -9.06 & 95.99 & 100.92 \\
\hline UACC-257 & 110.22 & 63.05 & 117.16 & -51.58 & 105.10 & 104.98 \\
\hline UACC-62 & 95.06 & 29.49 & 92.34 & -48.85 & 90.84 & 99.15 \\
\hline \multicolumn{7}{|l|}{ Ovarian Cancer } \\
\hline IGROV1 & 66.52 & 6.46 & 100.30 & -55.93 & 87.58 & 92.79 \\
\hline
\end{tabular}




\begin{tabular}{|c|c|c|c|c|c|c|}
\hline OVCAR-3 & 95.64 & 38.73 & 103.69 & -81.47 & 100.85 & 105.06 \\
\hline OVCAR-4 & 109.09 & 25.25 & 96.41 & -59.70 & 118.91 & 108.12 \\
\hline OVCAR-5 & 98.36 & 66.42 & 109.93 & 10.35 & 95.98 & 98.17 \\
\hline OVCAR-8 & 98.74 & 14.44 & 108.14 & -23.54 & 101.28 & 104.82 \\
\hline NCI/ADR-RES & 94.30 & 14.91 & 98.53 & -28.90 & 97.97 & 96.44 \\
\hline SK-OV-3 & 94.09 & 43.99 & 102.85 & -32.58 & 102.10 & 109.02 \\
\hline \multicolumn{7}{|l|}{ Renal Cancer } \\
\hline $786-0$ & 105.89 & 12.73 & 106.72 & -63.21 & 115.76 & 101.79 \\
\hline $\mathrm{ACHN}$ & 92.74 & 6.58 & 106.51 & -54.02 & 96.81 & 97.34 \\
\hline CAKI-1 & 94.26 & -2.66 & 94.15 & -71.98 & 96.09 & 100.02 \\
\hline RXF 393 & 101.14 & -10.80 & 124.22 & -76.09 & 109.09 & 110.90 \\
\hline SN12C & 89.83 & -4.86 & 102.59 & -75.38 & 89.90 & 99.10 \\
\hline TK-10 & 129.41 & 46.95 & 97.55 & -38.99 & 109.69 & 97.50 \\
\hline UO-31 & 82.98 & -11.02 & 85.09 & -65.01 & 88.43 & 83.69 \\
\hline \multicolumn{7}{|l|}{ Prostate Cancer } \\
\hline $\mathrm{PC}-3$ & 99.39 & 2.45 & 108.80 & -60.51 & 104.23 & 105.00 \\
\hline DU-145 & 102.77 & -58.73 & 108.34 & -38.24 & 102.72 & 101.53 \\
\hline \multicolumn{7}{|l|}{ Breast Cancer } \\
\hline MCF7 & 86.18 & 12.27 & 97.03 & -35.19 & 101.74 & 93.26 \\
\hline MDA-MB-231/ATCC & 98.38 & -6.31 & 99.23 & -8.73 & 92.92 & 90.37 \\
\hline HS 578T & 93.67 & 32.36 & 113.15 & -21.93 & 110.10 & 107.27 \\
\hline BT-549 & 102.88 & 32.74 & 110.39 & -25.44 & 109.55 & 105.52 \\
\hline $\mathrm{T}-47 \mathrm{D}$ & 97.46 & 11.86 & 77.24 & -34.25 & 95.71 & 95.97 \\
\hline MDA-MB-468 & 94.12 & -23.13 & 126.84 & -11.52 & 98.25 & 99.39 \\
\hline
\end{tabular}

ND: Not determined

In the present investigation, compounds $\mathbf{3 a}, \mathbf{3 c}, \mathbf{4 b}$ and $\mathbf{4 c}$ (Table 1) displayed modest potency against the tested tumor cell lines and were considered to be the least effective members. By contrast, compound $\mathbf{3 d}$ proved to be lethal to the majority of the cancer lines. The cytotoxic effect of compound 3d was apparent across all tumor cell lines tested, except leukemia, with 47 out of the 52 non-leukemic cell lines showing negative growth percentages. Also, strong lethality was induced by this compound against leukemia cancer cell lines RPMI-8226 and K-562 where the G\% were $6.40 \%$ and $14.32 \%$, respectively. Similarly, a complete cell death is achieved by compound $\mathbf{3 b}$ against leukemia cancer cell line MOLT-4, non-small cell lung cancer cell lines NCIH226, NCI-H460, CNS cancer cell line U251, melanoma cancer cell line MALME-3M, ovarian cancer cell line OVCAR-3, renal cancer cell lines CAKI-1, RXF-393, SN12C, UO-31, prostate cancer cell line DU-145, breast cancer cell lines MDA-MB-231/ATCC and MDA-MB-468. However, this compound elicited considerably greater growth inhibition activity against many cancer cell lines such as leukemia cancer cell line HL- 
60(TB), K-562, non-small cell lung cancer cell line HOP-62,NCI-H522, colon cancer cell line SW-620, CNS cancer cell line SF-268, melanoma cancer cell line SK-MEL-2, ovarian cancer cell line IGROV1, renal cancer cell line ACHN, prostate cancer cell line PC-3 and breast cancer cell line T-47D, where the G\% were 8.93\%, 10.70\%, 8.69\%, $7.58 \%, 7.11 \%, 9.40 \%, 11.80 \%, 6.46 \%, 6.58 \%, 2.45 \%$ and $11.86 \%$, respectively.

From the above mentioned results, it is obvious that compound $\mathbf{4 c}$ is considered to be the least effective against the tested tumor cell lines. However, it achieved potent selectivity and inhibition of Ets-1 phosphorylation by ERK2. The matter that may be attributed to, when BRAF- mutant A375 melanoma cell lines been treated with compound $\mathbf{4 c}$, the most potent derivative, it did not exhibit any effect on the MAP kinase pathway, which can be the reason behind its null effect on the NCI cytotoxicity studies. Given its potency towards ERK activity in-vitro. So, further structure improvement is recommended to enhance its ability to get into the cells for improving its cellular activities and pharmacokinetic profile.

\subsubsection{In vitro five-dose NCI 60 cell panel assay}

Interestingly, compounds $\mathbf{3 b}$ and $\mathbf{3 d}$ exhibited the most pronounced inhibitory activity in single dose preliminary tests, so they were progressed to further advanced five dose testing. The cytotoxic and/or growth inhibitory effects of the compounds were quantified in vitro against the full NCI-60 panel of human tumor cell lines using a series of five 10fold dilutions, ranging from $10^{-4} \mathrm{M}$ to $10^{-8} \mathrm{M}$. Three dose response parameters were calculated for each cell line, $\mathrm{GI}_{50}$ (molar concentration required for $50 \%$ growth inhibition), TGI (molar concentration leading to total growth inhibition), and $\mathrm{LC}_{50}$ (molar concentration resulting in 50\% cell death) (Tables 2 and 3). 
Figure 2. Growth inhibitory activity of compound 3d at different dose levels against the NCI-60 cell lines, grouped into the nine different cancer types included in the panel.

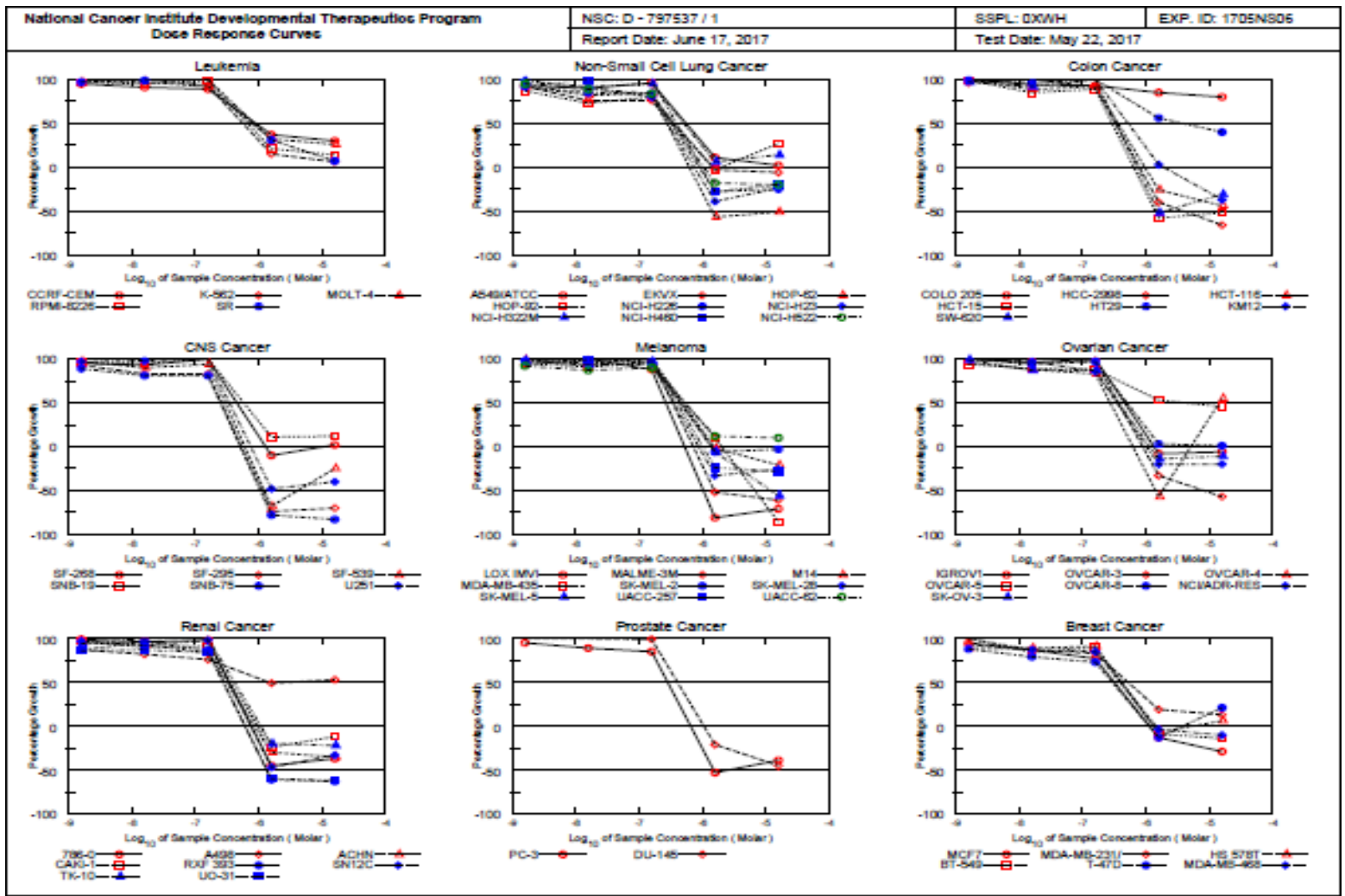

Figure 3. Growth inhibitory activity of compound $\mathbf{3 b}$ at different dose levels against the

NCI-60 cell lines, grouped into the nine different cancer types included in the panel

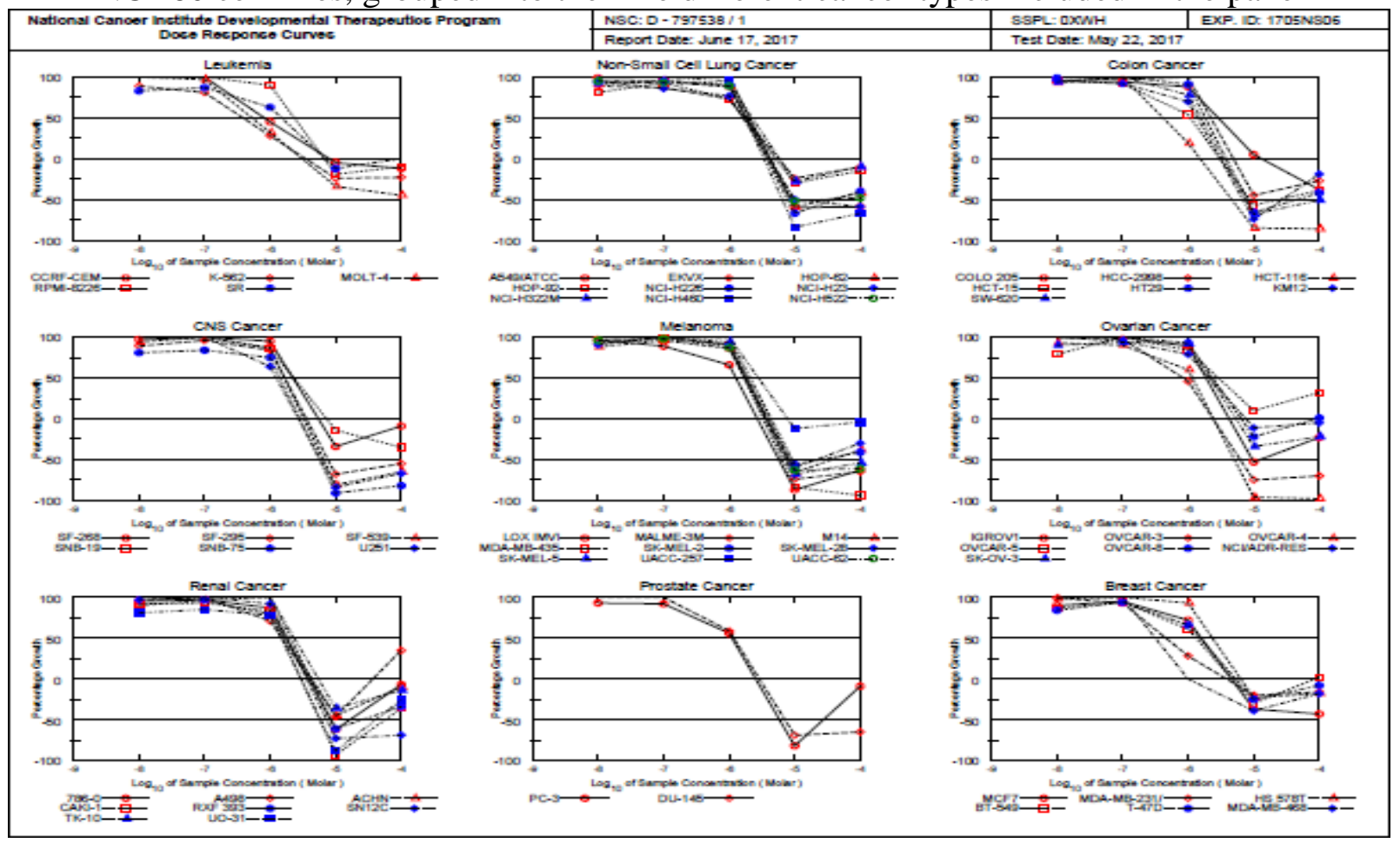


Table 2. NCI cytotoxic parameters (GI $\mathrm{G}_{50}$, TGI and $\mathrm{LC}_{50}$ ) of compound $\mathbf{3 d}$ in $\mu \mathrm{M}$.

\begin{tabular}{|c|c|c|c|}
\hline \multicolumn{4}{|c|}{ Subpanel tumor cell lines } \\
\hline & GI 50 & TGI & $\mathrm{LC}_{50}$ \\
\hline \multicolumn{4}{|l|}{ Leukemia } \\
\hline CCRF-CEM & 0.93 & $>16.7$ & 16.7 \\
\hline HL-60 (TB) & ND & ND & ND \\
\hline $\mathrm{K}-562$ & 0.59 & $>16.7$ & 16.7 \\
\hline MOLT-4 & 0.82 & 16.7 & 16.7 \\
\hline RPMI-8226 & 0.71 & 16.7 & 16.7 \\
\hline SR & 0.93 & 16.7 & 16.7 \\
\hline \multicolumn{4}{|c|}{ Non-Small Cell Lung Cancer } \\
\hline A549/ATCC & 0.58 & 16.7 & 16.7 \\
\hline EKVX & 0.35 & 1.53 & 16.7 \\
\hline HOP-62 & 0.29 & 0.66 & 1.50 \\
\hline HOP-92 & 0.38 & ND & 16.7 \\
\hline NCI-H226 & 0.34 & 0.94 & 16.7 \\
\hline NCI-H23 & 0.30 & 0.79 & 16.7 \\
\hline NCI-H322M & 0.53 & 16.7 & 16.7 \\
\hline NCI-H460 & 0.42 & 1.02 & 16.7 \\
\hline NCI-H522 & 0.36 & 1.11 & 16.7 \\
\hline \multicolumn{4}{|l|}{ Colon Cancer } \\
\hline COLO 205 & 16.7 & 16.7 & 16.7 \\
\hline HCC-2998 & 0.35 & 0.83 & 4.14 \\
\hline HCT-116 & 0.38 & 1.00 & 16.7 \\
\hline HCT-15 & 0.31 & 0.67 & 1.47 \\
\hline HT29 & 3.87 & 16.7 & 16.7 \\
\hline KM 12 & 0.57 & 1.97 & 16.7 \\
\hline SW-620 & 0.35 & 0.76 & ND \\
\hline \multicolumn{4}{|l|}{ CNS Cancer } \\
\hline SF-268 & 0.47 & ND & 16.7 \\
\hline SF-295 & 0.27 & 0.57 & 1.18 \\
\hline SF-539 & 0.31 & 0.64 & ND \\
\hline SNB-19 & 0.61 & 16.7 & 16.7 \\
\hline SNB-75 & 0.26 & 0.54 & 1.11 \\
\hline $\mathrm{U} 251$ & 0.37 & 0.80 & 16.7 \\
\hline \multicolumn{4}{|l|}{ Melanoma } \\
\hline LOX IMVI & 0.28 & 0.55 & 1.09 \\
\hline MALME-3M & 0.37 & 0.77 & 1.62 \\
\hline M14 & 0.52 & 1.60 & 16.7 \\
\hline MDA-MB-435 & 0.57 & 2.11 & 7.07 \\
\hline SK-MEL-2 & 0.47 & 1.46 & 16.7 \\
\hline SK-MEL-28 & 0.38 & 0.93 & 16.7 \\
\hline SK-MEL-5 & 0.48 & 1.47 & 12.8 \\
\hline UACC-257 & 0.48 & 1.11 & 16.7 \\
\hline UACC-62 & 0.55 & 16.7 & 16.7 \\
\hline \multicolumn{4}{|l|}{ Ovarian Cancer } \\
\hline IGROV1 & 0.49 & 1.41 & 16.7 \\
\hline OVCAR-3 & 0.39 & 0.93 & 8.68 \\
\hline OVCAR-4 & ND & ND & ND \\
\hline OVCAR-5 & 4.19 & 16.7 & 16.7 \\
\hline OVCAR-8 & 0.53 & 16.7 & 16.7 \\
\hline NCI/ADR-RES & 0.36 & 1.08 & 16.7 \\
\hline SK-OV-3 & 0.39 & 1.22 & 16.7 \\
\hline \multicolumn{4}{|l|}{ Renal Cancer } \\
\hline $786-0$ & 0.36 & 0.81 & 16.7 \\
\hline A498 & $\mathrm{ND}$ & 16.7 & 16.7 \\
\hline $\mathrm{ACHN}$ & 0.33 & 0.91 & 16.7 \\
\hline CAKI-1 & 0.38 & 1.03 & 16.7 \\
\hline RXF 393 & 0.30 & 0.65 & 1.41 \\
\hline SN12C & 0.36 & 0.79 & 16.7 \\
\hline TK-10 & 0.48 & 1.18 & 16.7 \\
\hline UO-31 & 0.29 & 0.64 & 1.41 \\
\hline \multicolumn{4}{|l|}{ Prostate Cancer } \\
\hline PC-3 & 0.30 & 0.69 & ND \\
\hline DU-145 & 0.43 & 1.11 & 16.7 \\
\hline \multicolumn{4}{|l|}{ Breast Cancer } \\
\hline MCF7 & 0.33 & 1.21 & 16.7 \\
\hline MDA-MB-231/ATCC & 0.54 & 16.7 & 16.7 \\
\hline
\end{tabular}




\begin{tabular}{llll} 
HS 578T & 0.44 & ND & 16.7 \\
BT-549 & 0.42 & 1.35 & 16.7 \\
T-47D & 0.31 & ND & 16.7 \\
MDA-MB-468 & 0.41 & 1.49 & 16.7 \\
\hline
\end{tabular}

ND: Not determined

Table 3. NCI cytotoxic parameters (GI $\mathrm{G}_{50}$, TGI and $\mathrm{LC}_{50}$ ) of compound $\mathbf{3 b}$ in $\mu \mathrm{M}$.

\begin{tabular}{|c|c|c|c|}
\hline Subpanel tumor cell lines & GI 50 & TGI & $\mathrm{LC}_{50}$ \\
\hline \multicolumn{4}{|l|}{ Leukemia } \\
\hline CCRF-CEM & 0.83 & 8.03 & $>100$ \\
\hline HL-60 (TB) & ND & ND & ND \\
\hline K-562 & 0.38 & 3.44 & $>100$ \\
\hline MOLT-4 & 0.53 & 3.02 & $>100$ \\
\hline RPMI-8226 & 2.32 & 6.68 & $>100$ \\
\hline SR & 1.49 & ND & $>100$ \\
\hline \multicolumn{4}{|c|}{ Non-Small Cell Lung Cancer } \\
\hline A549/ATCC & 2.10 & 4.26 & 8.64 \\
\hline EKVX & 1.72 & 5.66 & $>100$ \\
\hline HOP-62 & 1.81 & 3.97 & ND \\
\hline HOP-92 & 1.66 & 5.10 & $>100$ \\
\hline NCI-H226 & 1.79 & 3.73 & ND \\
\hline NCI-H23 & 1.63 & 4.04 & 10.4 \\
\hline NCI-H322M & 2.24 & 5.85 & $>100$ \\
\hline NCI-H460 & 1.78 & 3.39 & 6.44 \\
\hline NCI-H522 & 1.88 & 4.25 & ND \\
\hline \multicolumn{4}{|l|}{ Colon Cancer } \\
\hline COLO 205 & 2.85 & 12.8 & $>100$ \\
\hline HCC-2998 & 2.30 & 4.97 & $>100$ \\
\hline HCT-116 & 0.42 & 1.53 & 4.60 \\
\hline HCT-15 & 1.08 & 3.05 & $>100$ \\
\hline HT29 & 1.83 & 3.80 & $>100$ \\
\hline KM 12 & 1.38 & 3.07 & $>100$ \\
\hline SW-620 & 1.56 & 3.44 & 7.58 \\
\hline \multicolumn{4}{|l|}{ CNS Cancer } \\
\hline SF-268 & 2.24 & 5.47 & $>100$ \\
\hline SF-295 & 1.67 & 3.55 & 7.58 \\
\hline SF-539 & 1.67 & 3.31 & 6.57 \\
\hline SNB-19 & 2.31 & 7.30 & $>100$ \\
\hline SNB-75 & 1.42 & 2.84 & 5.68 \\
\hline $\mathrm{U} 251$ & 1.24 & 2.70 & 5.88 \\
\hline \multicolumn{4}{|l|}{ Melanoma } \\
\hline LOX IMVI & 1.28 & 2.71 & 5.76 \\
\hline MALME-3M & 1.76 & 3.54 & 7.11 \\
\hline M14 & 1.73 & 3.70 & ND \\
\hline MDA-MB-435 & 1.69 & 3.29 & 6.39 \\
\hline SK-MEL-2 & 2.12 & 4.40 & ND \\
\hline SK-MEL-28 & 1.87 & 4.00 & ND \\
\hline SK-MEL-5 & 1.87 & 3.81 & 7.73 \\
\hline UACC- 257 & 3.04 & 7.98 & $>100$ \\
\hline UACC-62 & 1.76 & 3.79 & 8.19 \\
\hline \multicolumn{4}{|l|}{ Ovarian Cancer } \\
\hline IGROV1 & 1.88 & 4.25 & ND \\
\hline OVCAR-3 & 0.83 & 2.40 & 6.24 \\
\hline OVCAR-4 & 1.17 & 2.43 & 5.06 \\
\hline OVCAR-5 & 2.87 & $>100$ & $>100$ \\
\hline OVCAR-8 & 2.38 & ND & $>100$ \\
\hline NCI/ADR-RES & 2.11 & 7.56 & $>100$ \\
\hline SK-OV-3 & 2.15 & 5.36 & $>100$ \\
\hline \multicolumn{4}{|l|}{ Renal Cancer } \\
\hline $786-0$ & 1.78 & 3.84 & ND \\
\hline A498 & 1.52 & ND & $>100$ \\
\hline $\mathrm{ACHN}$ & 1.72 & 4.36 & $>100$ \\
\hline CAKI-1 & 1.54 & 2.95 & ND \\
\hline RXF 393 & 2.04 & 4.17 & ND \\
\hline SN12C & 1.80 & 3.63 & 7.29 \\
\hline TK-10 & 2.91 & 5.97 & $>100$ \\
\hline
\end{tabular}




\begin{tabular}{lccc}
\hline UO-31 & 1.48 & 2.95 & ND \\
Prostate Cancer & & & \\
PC-3 & 1.11 & 2.55 & ND \\
DU-145 & 1.18 & 2.90 & 7.11 \\
Breast Cancer & & & \\
MCF7 & 1.59 & 4.58 & $>100$ \\
MDA-MB-231/ATCC & 0.46 & 3.86 & $>100$ \\
HS 578T & 2.34 & 6.33 & $>100$ \\
BT-549 & 1.33 & ND & $>100$ \\
T-47D & 1.48 & 5.26 & $>100$ \\
MDA-MB-468 & 0.31 & 0.99 &
\end{tabular}

ND: Not determined

\subsection{Chemistry}

\subsubsection{Material and Methods}

Melting points were taken in open capillaries on a Gallenkamp melting point apparatus (Weiss-Gallenkamp, Loughborough, UK) and are uncorrected. The IR spectra were recorded from potassium bromide disks with a FT device, Minia University. Elemental analyses were carried out at the Microanalytical Center, Cairo University, Egypt. The NMR spectra were measured in DMSO- $d_{6}$ on a Bruker AV-400 spectrometer $(400 \mathrm{MHz}$ for ${ }^{1} \mathrm{H}, 100 \mathrm{MHz}$ for ${ }^{13} \mathrm{C}$, and $40.55 \mathrm{MHz}$ for ${ }^{15} \mathrm{~N}$ ); and the chemical shifts are expressed in $\delta(\mathrm{ppm})$, versus internal tetramethylsilane $(\mathrm{TMS})=0$ for ${ }^{1} \mathrm{H}$ and ${ }^{13} \mathrm{C}$, and external liquid ammonia $=0$ for ${ }^{15} \mathrm{~N}$. Coupling constants are stated in Hz. Correlations were established using ${ }^{1} \mathrm{H}-{ }^{1} \mathrm{H}$ COSY, and ${ }^{1} \mathrm{H}_{-}{ }^{13} \mathrm{C}$ and ${ }^{1} \mathrm{H}-{ }^{15} \mathrm{~N}$ HSQC and HMBC experiments. Mass spectra were recorded on a Finnigan Fab $70 \mathrm{eV}$, Institute of Organic Chemistry, Karlsruhe University, Karlsruhe, Germany. TLC was performed on analytical Merck 9385 silica aluminum sheets (Kieselgel 60) with $\mathrm{Pf}_{254}$ indicator; TLC's were viewed at $\lambda_{\max }=254 \mathrm{~nm}$.

\subsubsection{Starting materials}

1,6-Disubstituted-quinoline-2,4-(1H,3H)-diones 1a-f were prepared according to the literature [32,33]. 1,4-Naphthoquinone (2) (Aldrich) was used as received.

\subsubsection{Products}


Reaction of 1a-f with 1,4-naphthoquinone (2)

A mixture of 4-hydroxy-2(1H)-quinolinones 1a-f $(1 \mathrm{mmol})$ and 1,4-naphthoquinone (2, $0.158 \mathrm{~g}, 1 \mathrm{mmol})$ in ethanol/DMF (1:1) containing $\mathrm{Et}_{3} \mathrm{~N}(0.5 \mathrm{~mL})$ was stirred at reflux temperature for 6-10 h. The reaction was monitored by TLC (until completion of the reaction). On cooling to room temperature, the precipitate was filtered off then washed with DMF ( 2 x $50 \mathrm{~mL}$ ). The filtrate containing 4a-c, was extracted by $\mathrm{CH}_{2} \mathrm{Cl}_{2}$, washed by cold water and the solvents were concentrated under vacuum. Compounds 3a-d and 4a-c were recrystallized from suitable solvents.

2,3-Bis(4-hydroxy-2-oxo-1,2-dihydroquinolin-3-yl)naphthalene-1,4-dione (3a). This compound was obtained as yellow crystals (DMF), $(0.315 \mathrm{~g}, 66 \%)$, mp. $350{ }^{\circ} \mathrm{C} . \mathrm{IR}(\mathrm{KBr})$ $\mathrm{v}=3330(\mathrm{NH}), 1725,1640(\mathrm{CO}), 1600,1585(\mathrm{Ar}-\mathrm{C}=\mathrm{C}) \mathrm{cm}^{-1}$; NMR (Table 4); MS

(FAB) m/z (\%) $477\left(\mathrm{M}^{+}, 20\right), 307$ (35), 289 (18), 155 (30), 154 (100), 137 (67), 107 (18); Anal. Calcd for $\mathrm{C}_{28} \mathrm{H}_{16} \mathrm{~N}_{2} \mathrm{O}_{6}$ (476.10) Calcd: C, 70.59; H, 3.38; N, 5.88. Found, C, 70.77; H, 3.35; N, 6.01 .

\section{2,3-Bis(4-hydroxy-6-methyl-2-oxo-1,2-dihydroquinolin-3-yl)naphthalene-1,4-dione}

(3b). This compound was obtained as yellow crystals (DMF/EtOH), (0.343 g, 68\%), mp. $355{ }^{\circ} \mathrm{C}$. IR (KBr) $\vee=3340(\mathrm{NH}), 1735,1625(\mathrm{CO}), 1605,1590(\mathrm{Ar}-\mathrm{C}=\mathrm{C}) \mathrm{cm}^{-1}$; NMR (Table 5; MS (FAB) m/z (\%) $505\left(\mathrm{M}^{+}, 30\right), 335$ (40), 154 (100), 107 (25). Anal. Calcd for $\mathrm{C}_{30} \mathrm{H}_{20} \mathrm{~N}_{2} \mathrm{O}_{6}$ (504.13) Calcd: C, 71.42; H, 4.00; N, 5.55. Found, C, 71.28; H, 3.95; N, 5.41 .

\section{2,3-Bis(6-chloro-4-hydroxy-2-oxo-1,2-dihydroquinolin-3-yl)naphthalene-1,4-dione}

(3c). This compound was obtained as violet crystals (DMF/EtOH), (0.349 g, 64\%), mp. 
$345{ }^{\circ} \mathrm{C} . \mathrm{IR}(\mathrm{KBr}) \vee=3320(\mathrm{NH}), 1725,1620(\mathrm{CO}), 1600,1585(\mathrm{Ar}-\mathrm{C}=\mathrm{C}) \mathrm{cm}^{-1} ;{ }^{1} \mathrm{H}$ NMR $\delta_{\mathrm{H}} 11.49(\mathrm{bs} ; 2 \mathrm{H}, \mathrm{NH}), 11.16(\mathrm{~b} ; 2 \mathrm{H}, \mathrm{OH}), 8.13\left(\mathrm{AA}^{\prime} \mathbf{X X}^{\prime}, J_{A X}=7.6, J_{A X^{\prime}}=1.4, J_{X X}=0\right.$; 2H, H-4'), $7.96\left(\mathbf{A A}^{\prime} X X, J_{A A}=7.0, J_{A X}=7.6, J_{A X^{\prime}}=1.4 ; 2 \mathrm{H}, \mathrm{H}-5^{\prime}\right), 7.77$ (d, $J=1.8 ; 2 \mathrm{H}$, H-5), 7.55 (dd, $J=8.8,1.8 ; 2 \mathrm{H}, \mathrm{H}-7), 7.18$ (d, $J=8.8 ; 2 \mathrm{H}, \mathrm{H}-8) ;{ }^{13} \mathrm{C}$ NMR $\delta_{\mathrm{C}} 182.24$ (C2'), 161.26 (C-2), 158.16 (C-4), 143.54 (C-1'), 136.82 (C-8a), 133.45 (C-5'), 132.47 (C3'), 130.81 (C-7), 125.92 (C-4'), 125.28 (C-6), 122.31 (C-5), 116.85 (C-8), 116.36 (C4ª), 106.08 (C-3); MS (FAB) m/z (\%) 543/545/546 (M+, 18-22), 460 (15), 318 (27), 307 (27), 289 (47), 274 (42), 154 (100), 137 (68), 107 (20). Anal. Calcd for $\mathrm{C}_{28} \mathrm{H}_{14} \mathrm{Cl}_{2} \mathrm{~N}_{2} \mathrm{O}_{6}$ (545.02) Calcd: C, 61.67; H, 2.59; N, 5.14. Found, C, 61.80; H, 2.41; N, 5.09.

\section{2,3-Bis(6-bromo-4-hydroxy-2-oxo-1,2-dihydroquinolin-3-yl)naphthalene-1,4-dione}

(3d). This compound was obtained as organge crystals (DMF), (0.444 g, 70\%), mp. 348 $350{ }^{\circ} \mathrm{C} . \mathrm{IR}(\mathrm{KBr}) \mathrm{v}=3325(\mathrm{NH}), 1715,1630(\mathrm{CO}), 1600,1595(\mathrm{Ar}-\mathrm{C}=\mathrm{C}) \mathrm{cm}^{-1} ;{ }^{1} \mathrm{H}$ NMR $\delta_{\mathrm{H}} 11.43(\mathrm{~s} ; 2 \mathrm{H}, \mathrm{NH}), 11.14(\mathrm{bs} ; 2 \mathrm{H}, \mathrm{OH}), 8.12\left(\mathrm{AA}^{\prime} \mathbf{X X}^{\prime}, J_{A A}=7.1, J_{A X}=7.4, J_{A X^{\prime}}=1.4\right.$; $\left.2 \mathrm{H}, \mathrm{H}-4^{\prime}\right), 7.88\left(\mathrm{AAXX}^{\prime}, J_{A X}=7.4, J_{A X^{\prime}}=1.4, J_{X X^{\prime}}=0 ; 2 \mathrm{H}, \mathrm{H}-5^{\prime}\right), 7.77(\mathrm{~d}, J=1.8 ; 2 \mathrm{H}$, $\mathrm{H}-5$ ), 7.56 (dd, $J=8.7,1.8 ; 2 \mathrm{H}, \mathrm{H}-7), 7.17$ (d, $J=8.7 ; 2 \mathrm{H}, \mathrm{H}-8) ;{ }^{13} \mathrm{C}$ NMR $\delta_{\mathrm{C}} 182.55$ (C2'), 161.09 (C-2), 159.11 (C-4), 143.76 (C-1'), 136.09 (C-8a), 133.48 (C-5'), 132.33 (C3'), 130.80 (C-7), 126.04 (C-4'), 125.97 (C-6), 121.98 (C-5), 116.88 (C-8), 116.40 (C4a), 105.87 (C-3); MS (FAB) m/z (\%) 633/634/636 (M+1 , 10-15), 307 (30), 289 (17), 154 (100), 137 (73), 136 (67), 107 (22). Anal. Calcd for $\mathrm{C}_{28} \mathrm{H}_{14} \mathrm{Br}_{2} \mathrm{~N}_{2} \mathrm{O}_{6}$ (631.92) Calcd: C, 53.02; H, 2.22; N, 4.42. Found, C, 53.17; H, 2.10; N, 4.58.

[Triethyl ammonium][2,3-bis(4-hydroxy-6-methyl-2-oxo-1,2-dihydroquinolin-3-yl)naphthalene-1,4-dione] (4a). This compound was obtained as red crystals (DMF/MeOH), (0.061 g, 10\%), mp. $>360{ }^{\circ} \mathrm{C} . \mathrm{IR}(\mathrm{KBr}) \vee=3210(\mathrm{NH}), 1679,1625(\mathrm{CO})$, 
1605, $1590\left(\right.$ Ar-C=C) $\mathrm{cm}^{-1}$, NMR (Table 6); MS (Fb) m/z (\%) $606\left(\mathrm{M}^{+}, 22\right), 504$ (30), 307 (21), 154 (40), 136 (21), 102 (100); Anal. Calcd for $\mathrm{C}_{36} \mathrm{H}_{35} \mathrm{~N}_{3} \mathrm{O}_{6}$ (605.25) Calcd: C, 71.39; H, 5.82; N, 6.94. Found, C, 71.54; H, 5.77; N, 7.12.

[Triethyl ammonium][2,3-bis(4-hydroxy-1-methyl-2-oxo-1,2-dihydroquinolin-3-yl)naphthalene-1,4-dione] (4b). This compound was obtained as brown crystals (DMF), (0.472 g, 78\%), mp. $>360{ }^{\circ} \mathrm{C} . \mathrm{IR}(\mathrm{KBr}) \vee=3190(\mathrm{NH}), 1676,1635(\mathrm{CO}), 1600,1585$ $(\mathrm{Ar}-\mathrm{C}=\mathrm{C}) \mathrm{cm}^{-1} ;{ }^{1} \mathrm{H}$ NMR $\delta_{\mathrm{H}} 8.9(\mathrm{~b} ; 2 \mathrm{H}, \mathrm{OH}), 8.03\left(\mathrm{AA}^{\prime} \mathbf{X X}^{\prime}, J_{A X}=7.7, J_{A X^{\prime}}=1.3, J_{X X^{\prime}}=\right.$ 0; 2H, H-4'), 8.01 (dd, $J=7.2,1.4 ; 2 \mathrm{H}, \mathrm{H}-5), 7.89\left(\mathbf{A A}^{\prime} X^{\prime}, J_{A A}=7.0, J_{A X}=7.7, J_{A X^{\prime}}=\right.$ 1.3; 2H, H-5'), 7.49 (dt, $\left.J_{d}=1.5, J_{t}=7.7 ; 2 \mathrm{H}, \mathrm{H}-7\right), 7.31(\mathrm{~d}, J=8.4 ; 2 \mathrm{H}, \mathrm{H}-8), 7.13(\mathrm{t}, J$ $=7.4 ; 2 \mathrm{H}, \mathrm{H}-6), 3.42(\mathrm{~s} ; 6 \mathrm{H}, \mathrm{H}-1 \mathrm{a}), 3.06\left(\mathrm{q}, J=7.3 ; 6 \mathrm{H}, \mathrm{Et}_{3} \mathrm{~N}\right), 1.14(\mathrm{t}, J=7.3 ; 9 \mathrm{H}$, $\left.\mathrm{Et}_{3} \mathrm{~N}\right) ;{ }^{13} \mathrm{C}$ NMR $\delta_{\mathrm{C}} 184.07$ (C-2'), $165.16(\mathrm{C}-4), 161.69$ (C-2), 145.76 (C-1'), 139.43 (C8a), 133.51 (C-5'), 132.66 (C-3'), 130.22 (C-7), 125.82 (C-4'), 124.75 (C-5), 120.41 (C4a), 120.32 (C-6), 113.56 (C-8), 105.44 (C-3), $45.71\left(\mathrm{Et}_{3} \mathrm{~N}\right), 28.15(\mathrm{C}-1 \mathrm{a}), 8.73\left(\mathrm{Et}_{3} \mathrm{~N}\right)$;

${ }^{15} \mathrm{~N}$ NMR $\delta_{\mathrm{N}} 130.8(\mathrm{~N}-1), 56.0\left(\mathrm{Et}_{3} \mathrm{~N}\right)$; MS (FAB) m/z (\%) $606\left(\mathrm{M}^{+}, 15\right), 504$ (19), 307 (15), 154 (35), 136 (27), 102 (100). Anal. Calcd for $\mathrm{C}_{36} \mathrm{H}_{35} \mathrm{~N}_{3} \mathrm{O}_{6}$ (605.25) Calcd: C, 71.39; H, 5.82; N, 6.94. Found, C, 71.53; H, 5.79; N, 7.11.

[Triethyl ammonium][2,3-bis(1-ethyl-4-hydroxy-2-oxo-1,2-dihydroquinolin-3-yl)naphthalene-1,4-dione (4c). This compound was obtained as red crystals (DMF/EtOH), (0.520 g, 82\%), mp. $320-321^{\circ} \mathrm{C} . \mathrm{IR}(\mathrm{KBr}) \mathrm{v}=3320(\mathrm{NH}), 1675,1620(\mathrm{CO}), 1610,1590$ $(\mathrm{Ar}-\mathrm{C}=\mathrm{C}) \mathrm{cm}^{-1} ;{ }^{1} \mathrm{H}$ NMR $\left(\mathrm{DMSO}_{-} \mathrm{d}_{6}\right) \delta_{\mathrm{H}}=1.04\left(\mathrm{ABX}_{3}, J_{A X}=J_{B X}=7.0 ; 3 \mathrm{H}, \mathrm{H}-1 \mathrm{~b}\right), 1.09$ $\left(\mathrm{t}, J=7.3 ; 9 \mathrm{H}, \mathrm{Et}_{3} \mathrm{~N}\right), 2.99\left(\mathrm{q}, J=7.2 ; 6 \mathrm{H}, \mathrm{Et}_{3} \mathrm{~N}\right), 3.99\left(\mathrm{ABX}_{3}, J_{A B}=14.3, J_{B X}=7.1 ; 2 \mathrm{H}\right.$, H-1a), $4.03\left(\mathrm{ABX}_{3}, J_{A B}=14.3, J_{A X}=7.1 ; 2 \mathrm{H}, \mathrm{H}-1 \mathrm{a}\right), 7.04$ (t, $\left.J=7.4 ; 2 \mathrm{H}, \mathrm{H}-6\right), 7.28$ (d, $J$ $=8.4 ; 2 \mathrm{H}, \mathrm{H}-8), 7.42\left(\mathrm{dt}, J_{d}=1.5, J_{t}=7.7 ; 2 \mathrm{H}, \mathrm{H}-7\right), 7.81\left(\mathbf{A A}^{\prime} X X^{\prime}, J_{A A^{\prime}}=7.2, J_{A X}=7.5\right.$, 
$\left.J_{A X^{\prime}}=1.5 ; 2 \mathrm{H}, \mathrm{H}-5^{\prime}\right), 7.95(\mathrm{dd}, J=7.7,1.3 ; 2 \mathrm{H}, \mathrm{H}-5), 7.96\left(\mathrm{AA}^{\prime} \mathbf{X X}^{\prime}, J_{A X}=7.5, J_{A X^{\prime}}=\right.$ $\left.1.5, J_{X X^{\prime}}=0 ; 2 \mathrm{H}, \mathrm{H}-4^{\prime}\right), 9.0 \mathrm{ppm}(\mathrm{b} ; 2 \mathrm{H}, \mathrm{OH}) ;{ }^{13} \mathrm{C}$ NMR $\left(\mathrm{DMSO}^{-} \mathrm{d}_{6}\right) \delta_{\mathrm{C}}=8.79\left(\mathrm{Et}_{3} \mathrm{~N}\right)$, 13.01 (C-1b), 35.36 (C-1a), $45.72\left(\mathrm{Et}_{3} \mathrm{~N}\right), 105.38$ (C-3), 113.34 (C-8), 120.09 (C-6), 120.51 (C-4a), 125.05 (C-5), 125.86 (C-4'), 130.89 (C-7), 132.64 (C-3') 133.45 (C-5'), 138.39 (C-8a), 145.79 (C-1'), 161.33 (C-2), 165.01 (C-4), 184.02 ppm (C-2'); ${ }^{15} \mathrm{~N}$ NMR $\left(\mathrm{DMSO}_{-} \mathrm{d}_{6}\right) \delta_{\mathrm{N}}=55.4\left(\mathrm{Et}_{3} \mathrm{~N}\right), 144.6 \mathrm{ppm}(\mathrm{N}-1) ; \mathrm{Ms}(\mathrm{Fab}) \mathrm{m} / \mathrm{z}(\%) 634(\mathrm{M}+, 18), 533$ (20), 307 (18), 154 (10), 102 (100). Anal. Calcd for $\mathrm{C}_{38} \mathrm{H}_{39} \mathrm{~N}_{3} \mathrm{O}_{6}$ (633.28): Calcd: C, 72.02; H, 6.20; N, 6.63. Found, C, 72.18; H, 6.34; N, 6.55.

\section{X-ray Crystal Structure Determinations of $4 c$ (include from the SI)}

The single-crystal X-ray diffraction study was carried out on a Bruker D8 Venture diffractometer with Photon100 detector at $123(2) \mathrm{K}$ using $\mathrm{Cu}-\mathrm{K} \alpha$ radiation $(\lambda=1.54178$ $\AA$ A. Direct Methods (SHELXS-97) [34] were used for structure solution and refinement was carried out using SHELXL-2014 (full-matrix least-squares on $F^{2}$ ) [35]. Hydrogen atoms were localized by difference electron density determination and refined using a riding model $(\mathrm{H}(\mathrm{O}, \mathrm{N})$ free $)$. A semi-empirical absorption correction was applied. The refinement with the listed atoms show residual electron density in two voids due to a heavily disordered water which could not be refined with split atoms. Therefore, the option "SQUEEZE" of the program package PLATON [36] was used to create a hkl file taking into account the residual electron density in the void areas. Therefore the atoms list and unit card do not agree (see cif-file for details).

4c: red crystals, $\mathrm{C}_{32} \mathrm{H}_{23} \mathrm{~N}_{2} \mathrm{O}_{6} \cdot \mathrm{C}_{6} \mathrm{H}_{10} \mathrm{~N} \cdot 0.5 \mathrm{H}_{2} \mathrm{O}, M_{\mathrm{r}}=642.73$, crystal size $0.18 \times 0.06$ $\times 0.03 \mathrm{~mm}$, monoclinic, space group $P 2{ }_{1} / \mathrm{n}$ (No. 14), $a=15.8124(5) \AA, b=$ 12.3817(4) $\AA, c=18.4717(6) \AA, \beta=107.089(2)^{\circ}, V=3456.8(2) \AA^{3}, Z=4, \rho=1.235$ 
$\mathrm{Mg} / \mathrm{m}^{-3}, \mu\left(\mathrm{Cu}-\mathrm{K}_{\alpha}\right)=0.687 \mathrm{~mm}^{-1}, F(000)=1364,2 \theta_{\max }=144.6^{\circ}, 28835$ reflections, of which 6783 were independent $\left(R_{\text {int }}=0.045\right), 430$ parameters, $R_{1}=0.062$ (for $5318 \mathrm{I}>$ $2 \sigma(\mathrm{I})), \mathrm{w} R_{2}=0.169$ (all data), $S=1.05$, largest diff. peak $/$ hole $=0.815$ (in the ammonium cation)/ -0.413 e $\AA^{-3}$. CCDC 1568166 (4c) contains the supplementary crystallographic data for this paper. These data can be obtained free of charge from The Cambridge Crystallographic Data Centre via www.ccdc.cam.ac.uk/data_request/cif. Due to the bad quality of the data of $2 \mathrm{a}-\mathrm{Br}$ (sb874) the data were not deposited with The Cambridge Crystallographic Data Centre.

\section{Computational Methodology}

Molecular docking into ERK2 receptor was carried out for the synthesized compounds as potential ERK2 inhibitors. All docking calculations were performed using Autodock4.2 software [37]. Receptor coordinates were taken from the crystal structure of ERK2 in complex with $N$-[(1S)-1-(3-Chloro-4-fluorophenyl) -2-hydroxyethyl]-2(tetrahydro-2H-pyran-4-yl-amino)-5,8-dihydropyrido[3,4-d]pyrimidine-7(6H)carboxamide (2SH) (PDB code: 4O6E [38]). The receptor was cleaned of water molecules, ions and the ligand $2 \mathrm{SH}$; as well the missing residues were constructed with MODELLER software [39]. The protonation state of the receptor was investigated using $\mathrm{H}++$ server [40]. AutoDock protocol [41] was then followed to prepare pdbqt file for ERK2 receptor. The related Autodock parameters were set as follows: 250 genetic algorithm (GA) runs, each with population size of 300, 25000000 energy evaluations, and a maximum of 27,000 generations per GA run. The docking grid was of dimension $70 \AA$ $\times 70 \AA \times 70 \AA$ with the spacing of $0.375 \AA$ and located at $16.20,7.50,12.40$ (XYZ 
coordinates). All examined compounds were energetically minimized using MMFF94S force field with the help of SZYBKI software [42]. Gasteiger atomic charges were assigned for the minimized compounds [43].

\section{Results and Discussion}

\subsection{Chemistry}

When a mixture of 2 equivalent of 4-hydroxy-2(1H)-quinolinones 1a-f and one equivalent| of 1,4-naphthoquinone (2) in ethanol/dimethylformamide (1:1) as a solvent and $0.5 \mathrm{ml} \mathrm{Et} 3 \mathrm{~N}^{\mathrm{N}}$ was stirred at reflux temperature for $6-10 \mathrm{~h}$, the bis-quinolinylquinones

3a-d and 4a-c were obtained (Scheme 1).

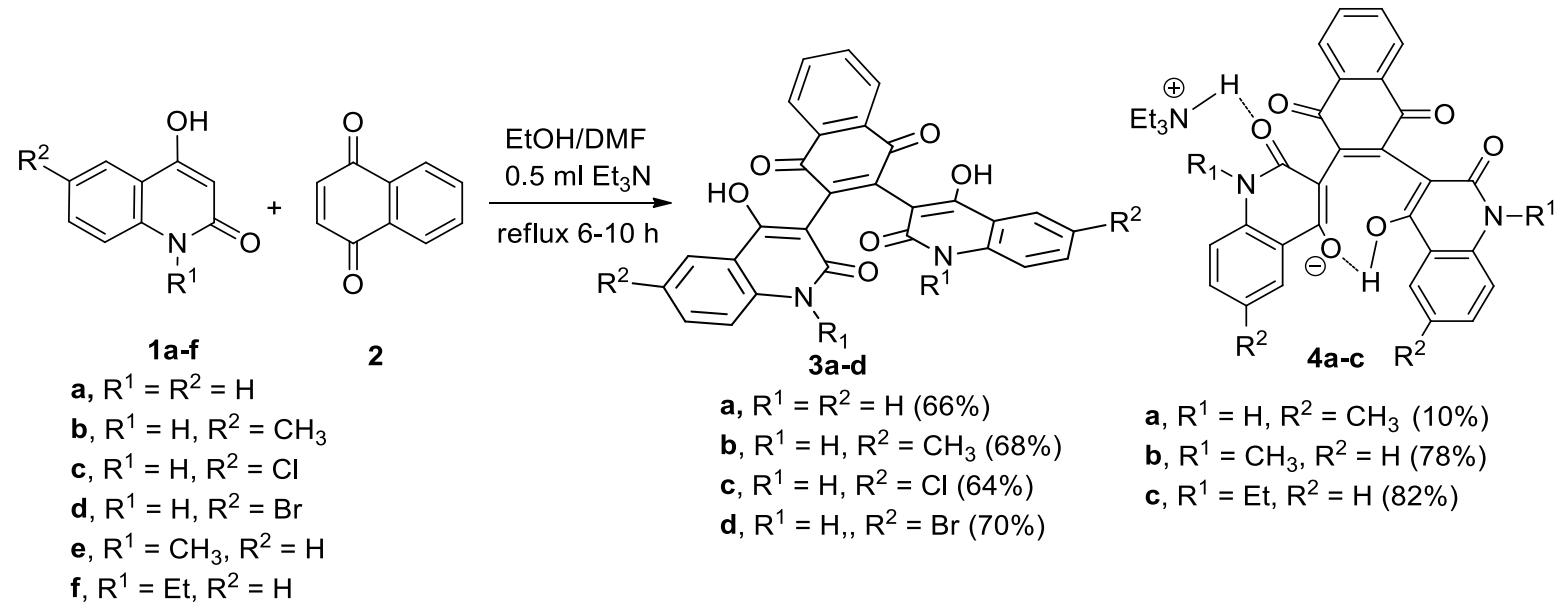

Scheme 1. Reaction of 4-hydroxy-2-quinlinones 1a-f with 1,4-naphthoquinone (2)

For example, compound 3a was obtained in $66 \%$ yield after recrystallization (see the experimental section and Table 4). The assignment of the structure of compounds 3a-d was confirmed by NMR analysis of the expected chemical shifts and 2D NMR spectra. In the ${ }^{1} \mathrm{H}$ NMR spectrum of $\mathbf{3 a}$, eight $2 \mathrm{H}$ signals were distinguished: two exchangeable singlets, a four-spin coupled system for the quinoline system, and an AA'XX' system for the naphthoquinone. The ${ }^{13} \mathrm{C}$ spectrum has 14 lines, consistent with either 3a or 3a' 
(Figure 7). The nitrogen atom resonates at $\delta_{N}=142.1 \mathrm{ppm}$; it gives HSQC correlation with a proton at $\delta_{H}=11.32 \mathrm{ppm}$, which therefore is assigned as the NH. This correlation leads to the assignment of quinolone tautomer 3a rather than 3a'. The nitrogen gives HMBC correlation to the doublet at $\delta_{H}=7.15 \mathrm{ppm}$, which is assigned as $\mathrm{H}-8$; its attached carbon resonates at $\delta_{C}=115.23 \mathrm{ppm}$. The NH gives strong HMBC correlation with C-8 and with a carbon at $\delta_{C}=105.42 \mathrm{ppm}$, assigned as C-3; these are both three-bond correlations.

Table 4. Spectroscopic data of compound 3a

${ }^{1} \mathrm{H}$ NMR:

$11.38(\mathrm{~s} ; 2 \mathrm{H})$

10.86 (bs; $2 \mathrm{H})$

$8.10\left(\mathrm{AA}^{\prime} \mathrm{XX} X^{\prime}, J_{\mathrm{AX}}=7.5, J_{\mathrm{AX}}=1.6, J_{\mathrm{XX}}{ }^{\prime}=0 ; 2 \mathrm{H}\right) 7.92$

$7.92\left(\mathbf{A A}^{\prime} \mathrm{XX}^{\prime}, J_{\mathrm{AA}^{\prime}}=7.4, J_{\mathrm{AX}}=7.5, J_{\mathrm{AX}}=1.6 ; 2 \mathrm{H}\right) 8.10$

$7.80(\mathrm{~d}, J=7.9 ; 2 \mathrm{H})$

$7.47\left(\mathrm{dt}, J_{\mathrm{d}}=1.2, J_{\mathrm{t}}=7.7 ; 2 \mathrm{H}\right)$

$7.15(\mathrm{~d}, J=8.4 ; 2 \mathrm{H})$

$7.14(\mathrm{t}, J=8.3 ; 2 \mathrm{H})$

${ }^{13}$ C NMR: HSQC

182.44

161.74

159.24

144.01

138.09

133.48

132.51

130.89

125.94

123.30

121.23

115.23

114.89

105.42

${ }^{15}$ N NMR:

143.1

$\begin{array}{ll}\text { HSQC: } & \text { HMBC: } \\ & 8.10 \\ & 11.38 \\ & 7.80,7.15 \\ & 11.38,7.80,7.47,7.14 \\ & 8.10 \\ 7.92 & 7.92 \\ & 7.80 \\ 7.47 & 7.92 \\ 8.10 & 7.47 \\ 7.80 & 7.15 \\ 7.14 & 11.38,7.47,7.14 \\ 7.15 & \\ & 11.38\end{array}$

HMBC:

8.10

11.38

$7.47,7.15,7.14$

$7.80,7.15,7.14$

$7.80,7.47,7.14$

$7.80,7.47,7.15$

Assignment:

C-2'

C-2

C-4

C-1'

C-8a

C-5'

C-3'

C-7

C-4'

C-5

C-6

C-8

C-4a

C-3
Assignment:

$\mathrm{NH}$

$\mathrm{OH}$

H-4'

H-5,

H-5

H-7

H-8

H-6

Calc:

187.0

163.8

162.3

147.9

135.9

134.8

137.9

127.9

130.2

126.4

124.0

120.3

126.8

98.1 
The NH also gives weak HMBC correlation with carbons at $\delta_{C}=161.74$ and $138.09 \mathrm{ppm}$, assigned as C-2 and C-8a respectively; these are both two-bond correlations. Also, C-8a gives $\mathrm{HMBC}$ correlation with all protons in the four-spin system except $\mathrm{H}-8$. In addition to other correlation with the triplet at $\delta_{H}=7.14 \mathrm{ppm}$, which assigned as $\mathrm{H}-6$; this is a three-bond correlation. The attached carbon appears at $\delta_{C}=121.23 \mathrm{ppm}$, and gives HMBC correlation back to H-8. The other doublet in the four-spin system (at $\delta_{H}=7.80$ ppm) must be $\mathrm{H}-5$ and the other triplet (at $\delta_{H}=7.47 \mathrm{ppm}$ ) must be $\mathrm{H}-7$; their attached carbons appear $\delta_{C}=123.30$ (C-5) and 130.89 ppm (C-7), respectively. C-5 gives HMBC correlation with $\mathrm{H}-7$, and $\mathrm{C}-7$ gives $\mathrm{HMBC}$ correlation with $\mathrm{H}-5$; these are three-bond correlations. H-5 and H-8 give HMBC correlation with a carbon at $\delta_{C}=159.24$ ppm, assigned based on chemical shift as $\mathrm{C}-4$ rather than $\mathrm{C}-4 \mathrm{a}$; the carbon at $\delta_{C}=114.89 \mathrm{ppm}$, is assigned as C-4a. Moreover, the other aromatic protons are the AA'XX' system of the naphthoquinone substructure. The downfield signal at $\delta_{H}=8.10$ gives $\mathrm{HMBC}$ correlation with the quinone carbonyl carbon at $\delta_{C}=182.44 \mathrm{ppm}$; accordingly, these protons are assigned as H-4' and the upfield signal at $\delta_{H}=7.92 \mathrm{ppm}$, is assigned as H-5'. The attached carbons appear at $\delta_{C}=125.94$ (C-4') and $133.48 \mathrm{ppm}\left(\mathrm{C}-5^{\prime}\right)$. A carbon at $\delta_{C}=$ 132.51 gives $\mathrm{HMBC}$ correlation with $\mathrm{H}-5$ ', and is assigned as $\mathrm{H}-3$ '. The remaining carbon at $\delta_{C}=144.01 \mathrm{ppm}$, is assigned as C-1'. The NMR spectroscopic data of 3a are shown in Table 4. 

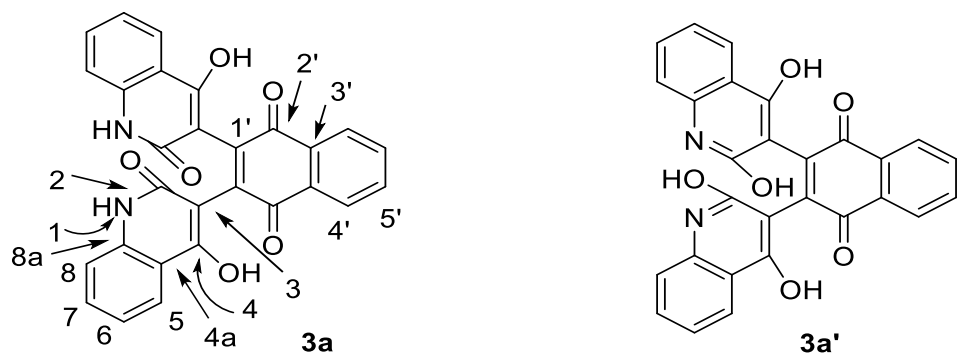

Figure 7. Alternative structure of $\mathbf{3 a}$, assigned as $\mathbf{3} \mathbf{a}$

In case of the reaction between $\mathbf{1 b}$ with $\mathbf{2}$, products $\mathbf{3 b}$ and $\mathbf{4 a}$ were separated (Scheme 1). Compound $\mathbf{3 b}$ was obtained as the major product (68\%), whereas salt $\mathbf{4 a}$ was in $10 \%$ yield percentage. Compound $\mathbf{3 b}$ (the distinctive carbons were assigned as shown in Figure 8) is the 1:2 adduct of 1,4-naphthoquinone (2) and 6-methylquinoline-2,4-dione (1b). The ${ }^{1} \mathrm{H}$ spectrum consists of two $6 \mathrm{H}$ methyl signals and seven $2 \mathrm{H}$ signals: two exchangeable singlets, a three-spin coupled system for the quinoline system, and an $\mathrm{AA}^{\prime} \mathrm{XX}$ ' system for the naphthoquinone. The ${ }^{13} \mathrm{C}$ spectrum has 15 lines, consistent with 3b. The assignment of a quinolone tautomer, rather than a quinolinol, rests on the observation of ${ }^{1} \mathrm{H}-{ }^{15} \mathrm{~N}$ HSQC correlation.

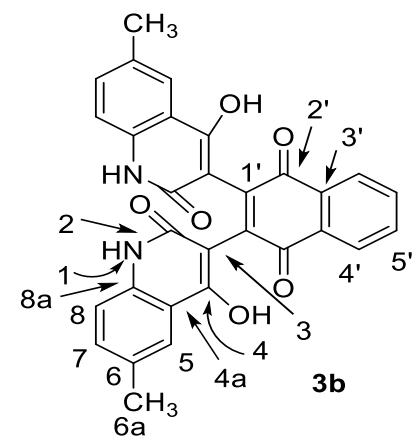

Figure 8. Distinctive carbons and nitrogen of compound $\mathbf{3 b}$

Table 5. Spectroscopic data of compound $\mathbf{3 b}$

${ }^{1}$ H NMR:

$11.32(\mathrm{bs} ; 2 \mathrm{H})$

$10.71(\mathrm{~b} ; 2 \mathrm{H})$

$8.10\left(\mathbf{A A}^{\prime} \mathrm{XX}^{\prime}, J_{\mathrm{AA}^{\prime}}=7.1, J_{\mathrm{AX}}=7.7, J_{\mathrm{AX}^{\prime}}=1.4 ; 2 \mathrm{H}\right) 7.93$

$7.93\left(\mathrm{AA}^{\prime} \mathrm{XX}^{\prime}, J_{\mathrm{AX}}=7.7, J_{\mathrm{AX}}=1.4, J_{\mathrm{XX}}{ }^{\prime}=0 ; 2 \mathrm{H}\right) \quad 8.10$
Assignment:

$\mathrm{NH}$

$\mathrm{OH}$

H-4'

H-5' 
7.60 (“s"; $2 \mathrm{H})$

$7.30(\mathrm{dd}, J=8.3,1.3 ; 2 \mathrm{H})$

$7.07(\mathrm{~d}, J=8.4 ; 2 \mathrm{H})$

$2.29(\mathrm{~s} ; 6 \mathrm{H})$

$\begin{array}{lllll}{ }^{13} \text { C NMR: } & \text { HSQC: } & \text { HMBC: } & \begin{array}{l}\text { Assignment: } \\ \text { Calc: }\end{array} \\ 182.47 & & & \text { C-2 } & 187.0 \\ 161.76 & & & \text { C-2 } & 163.8 \\ 159.04 & & 7.60,7.07 & \text { C-4 } & 162.8 \\ 144.14 & & & \text { C-1' } & 147.9 \\ 136.05 & & 7.60,7.30 & \text { C-8a } & 132.9 \\ 133.52 & 7.93 & 8.10 & \text { C-5 } & 134.8 \\ 132.48 & 7.30 & & \text { C-7 } & 128.6 \\ 130.34 & & 7.07,2.29 & \text { C-6 } & 133.2 \\ 125.96 & 8.10 & & \text { C-4 } & 130.2 \\ 122.72 & 7.60 & 2.29 & \text { C-5 } & 127.1 \\ 115.11 & & & \text { C-4a } & 126.7 \\ 114.89 & 7.07 & 11.32 & \text { C-8 } & 120.2 \\ 105.50 & & 11.32 & \text { C-3 } & 98.1 \\ 20.49 & 2.29 & & \text { C-6a } & 21.3\end{array}$

${ }^{15}$ N NMR: HSQC: HMBC: Assignment: $142.1 \quad 11.32$

$\begin{array}{ll}7.30 & \text { H-5 } \\ 7.60,7.07 & \text { H-7 } \\ 7.30 & \text { H-8 } \\ 7.60,7.30 & \text { H-6a }\end{array}$

H-6a 
the NH. This proton gives HMBC correlation with one or both of $\mathrm{C}-8$ and the carbon at $\delta_{C} 115.11$; based partly on this correlation and partly on chemical shift, this carbon is assigned as $\mathrm{C}-4 \mathrm{a}$. The $\mathrm{NH}$ also gives $\mathrm{HMBC}$ correlation with a carbon at $\delta_{C} 105.50$, assigned as C-3. The other aromatic protons are the AA'XX' system of the naphthoquinone substructure. On chemical-shift grounds, the downfield signal at $\delta_{H} 8.10$ is assigned as H-4' and the upfield signal at $\delta_{H} 7.93$ is assigned as $\mathrm{H}-5$ '; the attached carbons appear at $\delta_{C} 125.96\left(\mathrm{C}^{\prime} 4^{\prime}\right)$ and $133.52\left(\mathrm{C}-5^{\prime}\right)$. A carbon at $\delta_{C} 132.12$ gives HMBC correlation with $\mathrm{H}-5^{\prime}$, and is assigned as H-3'. The remaining carbons at $\delta_{C}$ 182.47, 161.76, and 144.14 are assigned, on chemical-shift grounds, as C-2', C-2, and C1' respectively.

Based on NMR spectroscopic data (Table 6), the structure of 4 a was defined as triethylammonium salt of 2,3-bis(quinolon-3-yl)naphthoquinone (Scheme 1). The triethyl amine salt was formed between triethyl amine molecule and the hydroxyl groups (Figure 9).

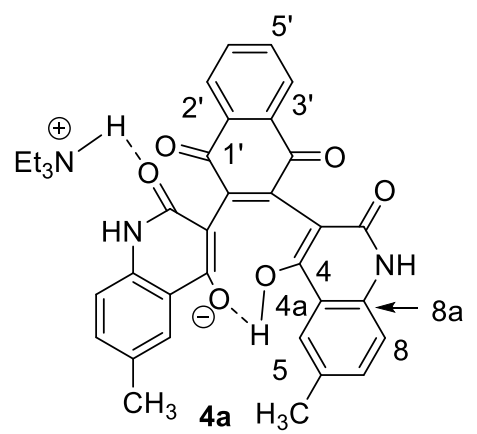

Figure 9. Structure of side product $\mathbf{4 a}$ obtained by the reaction of $\mathbf{1 b}$ with $\mathbf{2}$

The ${ }^{1} \mathrm{H}$ spectrum consists of one $6 \mathrm{H}$ methyl signal and seven $2 \mathrm{H}$ signals: two exchangeable singlets, a three-spin coupled system for the quinoline system, and an $\mathrm{AA}^{\prime} \mathrm{XX}$ ' system for the naphthoquinone. An equivalent of triethylamine is present. The ${ }^{13} \mathrm{C}$ spectrum has 17 lines, consistent with a triethylammonium salt of $4 \mathbf{a}$. The ${ }^{1} \mathrm{H}_{-}{ }^{15} \mathrm{~N}$ 
HSQC correlation observed in sample $\mathbf{4 a}$ is absent, but the nitrogen has the same hybridization, so the compound is assigned as a quinolone not a quinolinol. We believe the triethylammonium ion to be associated with the hydroxyl groups not the nitrogens, because C-4 resonates over $7 \mathrm{ppm}$ downfield of its position in $\mathbf{4 a}$, while the chemical shift of $\mathrm{N}-1$ changes by less than $1 \mathrm{ppm}$. The methyl protons and the three-spin system of the 6-substituted quinoline substructure are distinctive at $\delta_{\mathrm{H}} 2.30(\mathrm{H}-6 \mathrm{a}), 7.65$ (apparent singlet, a doublet with a small $J$; H-5), 7.17 (dd; H-7), and 7.02 (doublet, large $J$; H-8) respectively; they give HSQC correlation with their respective attached carbons at $\delta_{\mathrm{C}}$ 20.55 (C-6a), $123.93(\mathrm{C}-5), 132.68(\mathrm{C}-7)$, and $114.34(\mathrm{C}-8)$. A carbon at $\delta_{\mathrm{C}} 129.71$ gives HMBC correlation with $\mathrm{H}-6 \mathrm{a}$ and $\mathrm{H}-8$, and is assigned as C-6; a carbon at $\delta_{\mathrm{C}} 136.59$ gives $\mathrm{HMBC}$ correlation with $\mathrm{H}-5$ and $\mathrm{H}-7$ and $\mathrm{H}-8$, and is assigned as $\mathrm{C}-8 \mathrm{a}$. A carbon at $\delta_{\mathrm{C}} 166.24$ gives $\mathrm{HMBC}$ correlation with $\mathrm{H}-5$ and $\mathrm{H}-8$, and is assigned as $\mathrm{C}-4$; this is over $7 \mathrm{ppm}$ downfield of its shift in $\mathbf{4 a}$. The only other carbon more than $2 \mathrm{ppm}$ from its position in $4 \mathbf{a}$ is $\mathrm{C}-4 \mathrm{a}$ at $\delta_{\mathrm{C}} 119.14$, shifted by $4 \mathrm{ppm}$. The nitrogen atom resonates at $\delta_{\mathrm{N}}$ 142.9, only 0.8 ppm downfield of its shift in $\mathbf{4 a}$; it does not give HSQC correlation, but gives $\mathrm{HMBC}$ correlation with $\mathrm{H}-8$. Also, $\mathrm{H}-8$ gives $\mathrm{HMBC}$ correlation with $\mathrm{C}-4 \mathrm{a}$. The carbon at $\delta_{\mathrm{C}} 105.90$ is assigned, based on chemical shift, as C-3. The other aromatic protons are the AA'XX' system of the naphthoquinone substructure. On chemical-shift grounds, the downfield signal at $\delta_{\mathrm{H}} 8.01$ is assigned as $\mathrm{H}-4^{\prime}$, and the upfield signal at $\delta_{\mathrm{H}}$ 7.87 is assigned as $\mathrm{H}-5^{\prime}$; the attached carbons appear at $\delta_{\mathrm{C}} 125.78$ (C-4') and 133.39 (C5'). A carbon at $\delta_{\mathrm{C}} 132.54$ gives $\mathrm{HMBC}$ correlation with $\mathrm{H}-5^{\prime}$ ', and is assigned as $\mathrm{H}-3$ '. The remaining carbons at $\delta_{\mathrm{C}} 183.98,162.66$, and 146.13 are assigned, on chemical-shift 
grounds, as C-2', C-2, and C-1' respectively; C-2' also gives $\mathrm{HMBC}$ correlation with $\mathrm{H}-$ $4^{\prime}$.

Table 6. Spectroscopic data of compound $\mathbf{4 a}$.

\begin{tabular}{|c|c|c|}
\hline $\begin{array}{l}{ }^{1} \mathbf{H} \text { NMR: } \\
10.56 \text { (bs; 2H) }\end{array}$ & I COSY: & $\begin{array}{c}\text { Assignment: } \\
\mathrm{NH}\end{array}$ \\
\hline $8.01\left(\mathrm{AA}^{\prime} \mathbf{X X}^{\prime}, J_{\mathrm{AX}}=7.7, J_{\mathrm{AX}}=1.4, J_{\mathrm{XX}}=0 ; 2 \mathrm{H}\right)$ & 7.87 & $\mathrm{H}-4$ \\
\hline $7.87\left(\mathbf{A A}^{\prime} \mathrm{XX}^{\prime}, J_{\mathrm{AA}^{\prime}}=7.0, J_{\mathrm{AX}}=7.7, J_{\mathrm{AX}^{\prime}}=1.4 ; 2 \mathrm{H}\right.$ & 8.01 & $\mathrm{H}-5$, \\
\hline 7.65 (“s”; $2 \mathrm{H})$ & $7.17,7.02,2.30$ & $\mathrm{H}-5$ \\
\hline $7.17(\mathrm{dd}, J=8.3,1.8 ; 2 \mathrm{H})$ & $7.65,7.02,2.30$ & $\mathrm{H}-7$ \\
\hline $7.02(\mathrm{~d}, J=8.2 ; 2 \mathrm{H})$ & $7.65,7.17,2.30$ & $\mathrm{H}-8$ \\
\hline $5.1(\mathrm{~b} ; 2 \mathrm{H})$ & & $\mathrm{OH}$ \\
\hline $3.01(\mathrm{q}, J=7.3 ; 6 \mathrm{H})$ & 1.11 & $\mathrm{Et}_{3} \mathrm{~N}$ \\
\hline $2.30(\mathrm{~s} ; 6 \mathrm{H})$ & $7.65,7.17,7.02$ & H-6a \\
\hline $1.11(\mathrm{t}, J=7.3 ; 9 \mathrm{H})$ & 3.01 & $\mathrm{Et}_{3} \mathrm{~N}$ \\
\hline
\end{tabular}

\begin{tabular}{|c|c|c|c|}
\hline $\begin{array}{l}\mathbf{1 5}_{\mathbf{N}} \text { NMR: } \\
142.9 \\
60.7\end{array}$ & HSQC: & $\begin{array}{l}\text { HMBC: } \\
7.02 \\
1.11\end{array}$ & $\begin{array}{l}\text { Assignment: } \\
\mathrm{N}-1 \\
\mathrm{Et}_{3} \mathrm{~N}\end{array}$ \\
\hline $\begin{array}{l}{ }^{13} \text { C NMR: } \\
183.98\end{array}$ & HSQC: & $\begin{array}{l}\text { HMBC: } \\
8.01\end{array}$ & $\begin{array}{l}\text { Assignment: } \\
\text { C-2, }\end{array}$ \\
\hline 166.24 & & $7.65,7.02$ & C-4 \\
\hline 162.66 & & & $\mathrm{C}-2$ \\
\hline 146.13 & & & $\mathrm{C}-1$, \\
\hline 136.59 & & $7.65,7.17,7.02$ & C-8a \\
\hline 133.39 & 7.87 & $8.01,7.87$ & C-5, \\
\hline 132.68 & 7.17 & & $\mathrm{C}-7$ \\
\hline 131.54 & & 7.87 & $\mathrm{C}-3^{\prime}$ \\
\hline 129.71 & & $7.02,2.30$ & C-6 \\
\hline 125.78 & 8.01 & 7.87 & C-4' \\
\hline 123.93 & 7.65 & 2.30 & C-5 \\
\hline 119.14 & & 7.02 & $C-4 a$ \\
\hline 114.34 & 7.02 & & C-8 \\
\hline 105.90 & & & C-3 \\
\hline 45.70 & 3.01 & $3.01,1.11$ & $\mathrm{Et}_{3} \mathrm{~N}$ \\
\hline 20.55 & 2.30 & 7.65 & $C-6 a$ \\
\hline 8.88 & 1.11 & 3.01 & $\mathrm{Et}_{3} \mathrm{~N}$ \\
\hline
\end{tabular}

Compound $\mathbf{4 c}$ was formed from the reaction of $\mathbf{1 f}$ with $\mathbf{2}$ and by the same way formed as in case of $\mathbf{4 a}$ and $\mathbf{4 b} . \mathrm{Et}_{3} \mathrm{~N}$ molecule is engaged in the salt with hydroxyl groups. 
Spectroscopic parameters appear in the Experimental Section; X-ray structural analysis proved the structure of $\mathbf{4 c}$ as shown in Figure 10.

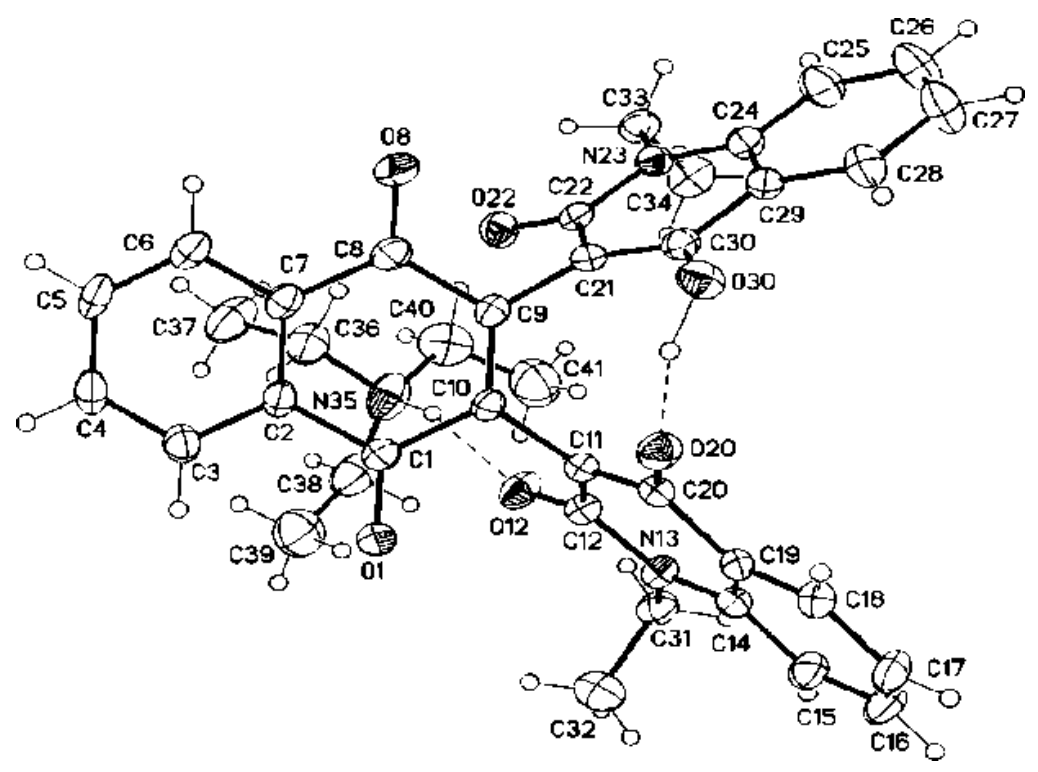

Figure 10. X-ray structural analysis of $\mathbf{4 c}$ (displacement parameters are drawn at 50\%

\subsection{Biology} probability level

As shown in Table 2, it is obvious that compound $\mathbf{3 d}$ exhibited marvelous cytotoxic activity against all tested human cancer cell lines with $\mathrm{GI}_{50}$ values ranging between $(0.26$ $-0.93 \mu \mathrm{M})$ except for colon cancer cell line $\mathrm{HT} 29\left(\mathrm{GI}_{50}=3.87 \mu \mathrm{M}\right)$, ovarian cancer cell line OVCAR-5 $\left(\mathrm{GI}_{50}=4.19 \mu \mathrm{M}\right)$ and colon cancer cell line COLO $205\left(\mathrm{GI}_{50}=16.7 \mu \mathrm{M}\right)$. Moreover, this compound is proved to be sensitive towards almost the tested cell lines, particularly non-small cell lung, CNS, melanoma and renal cancer cell lines where they showed the highest growth inhibitory activity. In the case of non-small cell lung cancer, CNS and renal cancer cell lines the average $\mathrm{GI}_{50}$ value was around $0.38 \mu \mathrm{M}$, while the $\mathrm{GI}_{50}$ value of melanoma cancer cell lines were around $0.45 \mu \mathrm{M}$. Meanwhile, the range of TGI value was $(0.45-16.7 \mu \mathrm{M})$. The $\mathrm{LC}_{50}$ values ranged between 1.09 and $16.7 \mu \mathrm{M}$. The cytotoxic effects were strongest in the non-small cell lung cancer line HOP-62 (1.50 
$\mu \mathrm{M})$, the colon cancer cell line HCT-15 (1.47 $\mu \mathrm{M})$, the CNS cancer lines SNB-75 (1.11 $\mu \mathrm{M})$ and SF-295 $(1.18 \mu \mathrm{M})$, the melanoma cancer cell line LOX IMVI (1.09 $\mu \mathrm{M})$ and the renal cancer lines RXF 393 and UO-31 (1.41 $\mu \mathrm{M})$.

The results recorded in Table 3 indicated that compound $\mathbf{3 b}$ demonstrated broad spectrum anti-tumor activity against the nine tumor sub-panels tested. It exhibited strong growth inhibitory activity against all the tested human cancer cell lines, with $\mathrm{GI}_{50}$ values ranged between $(0.31-3.04 \mu \mathrm{M})$ and the TGI values ranged between $(0.99-12.8 \mu \mathrm{M})$. A noticeable sensitivity profile was observed for leukemia cancer cell lines $\left(\mathrm{GI}_{50}\right.$ values ranging from $0.38-2.32 \mu \mathrm{M})$. Furthermore, this compound achieved a highly active growth inhibitory activity against non-small cell lung, colon, CNS, melanoma, ovarian and renal cancers, where an almost similar average $\mathrm{GI}_{50}$ ranging from (1.63- $\left.1.92 \mu \mathrm{M}\right)$ were recorded. Similarly, the tested compound experienced remarkable sensitivity towards prostate and breast cancer cell lines, where the average $\mathrm{GI}_{50}$ was around $1.2 \mu \mathrm{M}$. Notably, the highest growth inhibitory activity was observed against the breast cancer cell line MDA-MB-468 withGI 50 value $0.31 \mu \mathrm{M}$ and the strongest cytotoxic effects were observed in the non-small cell lung cancer lines NCI-H460 $\left(\mathrm{LC}_{50}=6.44 \mu \mathrm{M}\right)$, A549/ATCC $\left(\mathrm{LC}_{50}=8.46 \mu \mathrm{M}\right)$ and NCI-H23 $\left(\mathrm{LC}_{50}=10.4 \mu \mathrm{M}\right)$, the colon cancer cell lines HCT-116 $\left(\mathrm{LC}_{50}=4.60 \mu \mathrm{M}\right), \mathrm{SW}-620\left(\mathrm{LC}_{50}=7.58 \mu \mathrm{M}\right)$, all CNS cancer lines except SF-268, SNB-19, the melanoma lines except UACC-257, the ovarian cancer lines OVCAR-4 $\left(\mathrm{LC}_{50}=5.06 \mu \mathrm{M}\right)$ and OVCAR-3 $\left(\mathrm{LC}_{50}=6.24 \mu \mathrm{M}\right)$, the renal cancer line $\mathrm{SN} 12 \mathrm{C}\left(\mathrm{LC}_{50}=7.29 \mu \mathrm{M}\right)$ and the prostate cancer line DU-145 $\left(\mathrm{LC}_{50}=7.11 \mu \mathrm{M}\right)$. 


\section{Conclusion}

In this paper, we shed the biologist towards the importance of the biological activity of 2quinolones. Many interesting classes of this type of compounds would be valuable for drug future discovery. Herein we have a success to synthesize and in good yields two novel series of 2,3-bis-quinolinylnaphthalene-1,4-diones and bis-quinlinone triethylammonium salts. Compounds $\mathbf{3 a}, \mathbf{3 b}$ and $\mathbf{4 c}$ inhibited ETS-1 phosphorylation by ERK2 in a dose dependent manner. Moreover, Compound 4c showed selectivity for ERK2 inhibition together with ATP-competitive inhibition which was revealed by its binding pattern in the ATP-binding site of ERK2 receptor. However, an additional structure optimization is required for enhancing its pharmacokinetic profile. Compound 3d exhibited marvelous cytotoxicity towards approximately all NCI tumor cell lines tested showing negative growth percentages. The cytotoxic effect of compound $\mathbf{3 d}$ was apparent towards approximately all NCI tumor cell lines tested showing negative growth percentages. Compound $\mathbf{3 b}$ and $\mathbf{3 d}$ demonstrated broad spectrum anti-tumor activity against the nine tumor sub-panels tested in NCI in vitro five dose testing with $\mathrm{GI}_{50}$ values ranging between $(0.31-3.04 \mu \mathrm{M})$, and $(0.26-0.93 \mu \mathrm{M})$, respectively and TGI values ranging between $(0.99-12.8 \mu \mathrm{M})$ and $(0.45-16.7 \mu \mathrm{M})$, respectively. Finally, the newly synthesized compounds represent good starting point for the development of new cytotoxic lead compounds.

\section{References}

1. R.P. Verma, Anti-cancer activities of 1,4-naphthoquinones, Anti-Cancer Agents Med. Chem. 6 (2006) 489-499. 
2. A.K. McClendon, N. Osheroff, DNA topoisomerase II, genotoxicity, and cancer, Mutation Res. 623 (2007) 83-97.

3. H.R. Lawrence, A. Kazi, Y. Luo, R. Kendig, Y. Ge, S. Jain, K. Daniel, D. Santiago, W.C. Guida, S.M. Sebti, Synthesis and biological evaluation of naphthoquinone analogs as a novel class of proteasome inhibitors, Bioorg. Med. Chem. 18 (2010) $5576-5592$.

4. V.K. Tandon, H.K. Maurya, A. Tripathi, G.B. ShivaKeshava, P.K. Shukla, P. Srivastava, D. Panda, 2,3-Disubstituted 1,4-naphthoquinones, $\quad$ 12Hbenzo $[b]$ phenothiazine-6,11-diones and related compounds: synthesis and biological evaluation as potential antiproliferative and antifungal agents, Eur. J. Med. Chem. 44 (2009) 1086-1092.

5. E.H.G, da Cruz, C. M. B. Hussene, G.G. Dias, E.B.T. Diogo, I.M.M. de Melo, B.L. Rodrigues, M.G. da Silva, W.O. Valenca, C.A. Camara, R.N. de Oliveira, Y.G. de Paiva, M.O.F. Goulart, B.C. Cavalcanti, C. Pessoa, E.N. da Silva Junior, 1,2,3Triazole-, arylamino- and thio-substituted 1,4-naphthoquinones: potent antitumor activity, electrochemical aspects, and bioisosteric replacement of C-ring-modified lapachones, Bioorg. Med. Chem. 22 (2014) 1608-1619.

6. H.R. Nasiri, M.G. Madej, R. Panisch, M. Lafontaine, J.W. Bats, C.R.D. Lancaster, H. Schwalbe, Design, synthesis, and biological testing of novel naphthoquinones as substrate-based inhibitors of the quinol/fumarate reductase from Wolinella succinogenes, J. Med. Chem. 56 (2013) 9530-9541. 
7. D. Bhasin, S.N. Chettiar, J.P. Etter, M. Mok, P.-K. Li, Anticancer activity and SAR studies of substituted 1,4-naphthoquinones, Bioorg. Med. Chem. 21 (2013) 46624669.

8. C.-K. Ryu, D.-H. Kim, The synthesis and antimicrobial activities of some 1,4naphthoquinones, Arch. Pharm. Res. 15 (1992) 263-268.

9. S.-T. Huang, H.-S. Kuo, C.-L. Hsiao, Y.-L. Lin, Efficient synthesis of 'redoxswitched' naphthoquinone thiol-crown ethers and their biological activity evaluation, Bioorg. Med. Chem. 10 (2002) 1947-1952.

10. V.K. Tandon, D.B. Yadav, R.V. Singh, M. Vaish, A.K. Chaturvedi, P.K. Shukla, Synthesis and biological evaluation of novel 1,4-naphthoquinone derivatives as antibacterial and antiviral agents, Bioorg. Med. Chem. Lett. 15 (2005) 3463-3466.

11. A.J.M. Da Silva, C.D. Buarque, F.V. Brito, L. Aurelian, L.F. Macedo, L.H. Malkas, R.J. Hickey, D.V.S. Lopes, F. Noel, Y.L.B. Murakami, N.M.V. Silva, P.A. Melo, R.R.B. Caruso, N.G. Castro, P.R.R. Costa, Synthesis and preliminary pharmacological evaluation of new ( \pm ) naphthoquinones structurally related to lapachol, Bioorg. Med. Chem. 10 (2002) 2731-2738.

12. V.K. Tandon, R.V. Singh, S. Rai, R.B. Chhor, Z.K. Khan, Synthesis and pharmacological studies of some 2-t-amino and 2,3-di-t-amino substituted 1,4naphthoquinones and related compounds, Boll. Chim. Farm. 141 (2002) 304-310.

13. V.K. Tandon, R.B. Chhor, R.V. Singh, S. Rai, D.B. Yadav, Design, synthesis and evaluation of novel 1,4-naphthoquinone derivatives as antifungal and anticancer agents, Bioorg. Med. Chem. Lett. 14 (2004) 1079-1083. 
14. V.K. Tandon, H.K. Maurya, N.N. Mishra, P.K. Shukla, Design, synthesis, and biological evaluation of novel nitrogen and sulfur containing hetero-1,4naphthoquinones as potential antifungal and antibacterial agents, Eur. J. Med. Chem. 44 (2009) 3130-3137.

15. K. Sasaki, H. Abe, F. Yoshizaki, In vitro antifungal activity of naphthoquinone derivatives, Biol. Pharm. Bull. 25 (2002) 669-670.

16. V.K. Tandon, D.B. Yadav, H.K. Maurya, A.K. Chaturvedi, P.K. Shukla, Design, synthesis and biological evaluation of 1,2,3-trisubstituted-1,4dihydrobenzo $[\mathrm{g}$ ]quinoxaline-5,10-diones and related compounds as antifungal and antibacterial agents, Bioorg. Med. Chem. 14 (2006) 6120-6126.

17. J.-C. Lien, L.-J. Huang, C.-M.Teng, J.-P. Wang, S.-C. Kuo, Synthesis of 2-alkoxy 1,4naphthoquinone derivatives as antiplatelet, antiinflammatory, and antiallergic agents, Chem. Pharm. Bull. 50 (2002) 672-674.

18. Y.-R. Jin, C.-K. Ryu, C.-K. Moon, M.-R. Cho, Y.-P. Yun, Inhibitory effects of J78, a newly synthesized 1,4-naphthoquinone derivative, on experimental thrombosis and platelet aggregation, Pharmacology 70 (2004) 195-200.

19. D.-Y. Yuk, C.-K. Ryu, J.-T. Hong, K.-H. Chung, W.-S. Kang, Y. Kim, H.-S. Yoo, M.K. Lee, C.-K. Lee, Y.-P. Yun, Antithrombotic and antiplatelet activities of 2-chloro-3[4-(ehtylcarboxy)-phenyl]-amino-1,4-naphthoquinone (NQ12), a newly synthesized 1,4-naphthoquinone derivative, Biochem. Pharmacol. 60 (2000) 1001-1008.

20. T.H. Porter, T. Kishi, H. Kishi, K. Folkers, Synthesis, enzyme inhibition, and antitumor activity of new 1,4-benzoquinone analogs of coenzyme Q10, Bioorg. Chem. 7 (1978) 333-350. 
21. P. Hradil, P. Krejčí, J. Hlaváč, I. Wiedermannová, A. Lyčka, V. Bertolasi, Synthesis, NMR spectra and X-ray data of chloro and dichloro derivatives of 3-hydroxy-2phenylquinolin-4(1H)-ones and their cytostatic activity, J. Heterocycl. Chem. 41 (2004) 375-379.

22. M. Soural, J. Hlaváč, P. Funk, P. Džubák, M. Hajdúch, 2-Phenylsubstituted-3hydroxyquinolin-4(1H)-one-carboxamides: structure-cytotoxic activity relationship study, ACS Comb. Sci. 13 (2011) 39-44.

23. M. Soural, J. Hlaváč, P. Hradil, I. Fryšová, M. Hajdúch, V. Bertolasi, M. Maloň, Synthesis and cytotoxic activity of substituted 2-phenyl-3-hydroxy-4(1H)quinolinones-7-carboxylic acids and their phenacyl esters, Eur. J. Med. Chem. 41 (2006) 467-474.

24. P. Krejčí, P. Hradil, J. Hlaváč, M. Hajdúch, Preparation of 2-phenyl-3hydroxyquinolin-4(1H)-ones for treatment of immune system and proliferative disorders, PCT Int. Appl. (2008) WO2008028427 A1 (Chem. Abstr. 148:331570).

25. J. Hashim, T. N. Glasnov, J. M. Kremsner, C. O. Kappe, Symmetrical bisquinolones via metal-catalyzed cross-coupling and homocoupling reactions, J. Org Chem. 71 (2006) 1707-1710.

26. G. Uray, A.-M. Kelterer, J. Hashim, T. N. Glasnov, C. O. Kappe, W. M. F. Fabian, Bisquinolones as chiral fluorophores - a combined experimental and computational study of absorption and emission characteristics, J. Mol. Struct. 929 (2009) 85-96.

27. S.-L. Zhang, Z.-S. Huang, L.-K. An, X.-Z. Bu, L. Ma, Y.-M. Li, A.S.C. Chan, L.-Q. 
$\mathrm{Gu}$, Synthesis of zwitterionic 4-hydroxycoumarin derivatives through a unique reaction of 4-hydroxycoumarins with $p$-benzoquinone and pyridine, Org. Lett. 6 (2004) 4853-4855. DOI: 10.1021/ol048109p

28. E.M. El-Sheref, A.A. Aly, A.-F.E. Mourad, A.B. Brown, S. Bräse, M.E.M. Bakheet, Synthesis of pyrano[3,2-c]quinoline-4-carboxylates and 2-(4-oxo-1,4-dihydroquinlin3-yl)fumarates, Chem. Pap. 72 (2018) 181-190.

29. A.A. Aly, E.M. El-Sheref, A.-F.E. Mourad, A.B. Brown, S. Bräse, M.E.M. Bakheet, M. Nieger, Synthesis of spiro[indoline-3,4'-pyrano[3,2-c]quinolone]-3'-carbonitriles, Monatsh. Chem. 149 (2018) 635-644.

30. G.M. Morris, R. Huey, W. Lindstrom, M.F. Sanner, R.K. Belew, D.S. Goodsell, A.J. Olson, AutoDock4 and AutoDockTools4: Automated docking with selective receptor flexibility, J. Comput. Chem. 30 (2009) 2785-2791.

31. J.F. Blake, J.J. Gaudino, J. De Meese, P. Mohr, M. Chicarelli, H. Tian, R. Garrey, A. Thomas, C.S. Siedem, M.B. Welch, G. Kolakowski, R. Kaus, M. Burkard, M. Martinson, H. Chen, B. Dean, D.A. Dudley, S.E. Gould, P. Pacheco, S. ShahidiLatham, W. Wang, K. West, J. Yin, J. Moffat, J.B. Schwarz, Discovery of 5,6,7,8tetrahydropyrido[3,4- $d]$ pyrimidine inhibitors of Erk2, Bioorg. Med. Chem. Lett. 24 (2014) 2635-2639.

32. W.-T. Gao, W.-D. Hou, M.-R. Zheng, L.-J. Tang, Clean and convenient one-pot synthesis of 4-hydroxycoumarin and 4-hydroxy-2-quinolinone derivatives, Synth. Commun. (2010) 40, 732-738.

33. T. Kappe, The 'pyrono route' to 4-hydroxy-2-quinolones and 4-hydroxy-2-pyridones, Farmaco 54 (1999) 309-315. 
34. G.M. Sheldrick, A short history of SHELX, Acta Crystallogr. A64 (2008) 112-122.

35. G.M. Sheldrick, Crystal structure refinement with SHELXL, Acta Crystallogr. C71 (2015) 3-8.

36. A.L. Spek, Structure validation in chemical crystallography, Acta Crystallogr. D65 (2009) 148-155.

37. G.M. Morris, R. Huey, W. Lindstrom, M.F. Sanner, R.K. Belew, D.S. Goodsell, A.J. Olson, AutoDock4 and AutoDockTools4: Automated docking with selective receptor flexibility, J. Comput. Chem., 30 (2009) 2785-2791.

38. J.F. Blake, J.J. Gaudino, J. De Meese, P. Mohr, M. Chicarelli, H. Tian, R. Garrey, A. Thomas, C.S. Siedem, M.B. Welch, G. Kolakowski, R. Kaus, M. Burkard, M. Martinson, H. Chen, B. Dean, D.A. Dudley, S.E. Gould, P. Pacheco, S. ShahidiLatham, W. Wang, K. West, J. Yin, J. Moffat, J.B. Schwarz, Discovery of 5,6,7,8tetrahydropyrido[3,4-d]pyrimidine inhibitors of Erk2, Bioorg. Med. Chem. Lett., 24 (2014) 2635-2639.

39. M.A. Martí-Renom, A.C. Stuart, A. Fiser, R. Sánchez, F.M. and, A. Šali, Comparative Protein Structure Modeling of Genes and Genomes, Annu. Rev. Biophys. Biomol. Struct., 29 (2000) 291-325.

40. J.C. Gordon, J.B. Myers, T. Folta, V. Shoja, L.S. Heath, A. Onufriev, H++: a server for estimating $\mathrm{pK}(\mathrm{a}) \mathrm{s}$ and adding missing hydrogens to macromolecules, Nucleic Acids Res., 33 (2005) W368-W371.

41. S. Forli, R. Huey, M.E. Pique, M.F. Sanner, D.S. Goodsell, A.J. Olson, Computational protein-ligand docking and virtual drug screening with the AutoDock suite, Nat. Protocols, 11 (2016) 905-919. 
42. SZYBKI, in, OpenEye Scientific Software, Santa Fe, NM, USA, 2016.

43. J. Gasteiger, M. Marsili, ITERATIVE PARTIAL EQUALIZATION OF ORBITAL ELECTRONEGATIVITY - A RAPID ACCESS TO ATOMIC CHARGES,

Tetrahedron, 36 (1980) 3219-3228.

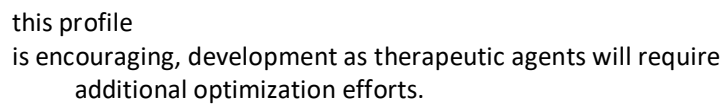
additional optimization efforts. 